\title{
Modelling Tuberculosis and Hepatitis B Co-infections
}

\author{
S. Bowong ${ }^{1,2,4, *}$ and J. Kurths $\mathbf{s}^{2,3}$ \\ ${ }^{1}$ Laboratory of Applied Mathematics, Department of Mathematics and Computer Science, \\ Faculty of Science, University of Douala, P.O. Box 24157 Douala, Cameroon \\ ${ }^{2}$ Postdam Institute for Climate Impact Research (PIK), \\ Telegraphenberg A 31, 14412 Potsdam, Germany \\ ${ }^{3}$ Department of Physics, Humboldt Universitat zu Berlin, 12489 Berlin, Germany \\ ${ }^{4}$ UMI 209 IRD/UPMC UMMISCO, Bondy, Projet MASAIE INRIA Grand Est, France \\ Projet Grimcape, LIRIMA, Cameroun
}

\begin{abstract}
Tuberculosis (TB) is the leading cause of death among individuals infected with the hepatitis B virus (HBV). The study of the joint dynamics of HBV and TB present formidable mathematical challenges due to the fact that the models of transmission are quite distinct. We formulate and analyze a deterministic mathematical model which incorporates of the co-dynamics of hepatitis B and tuberculosis. Two sub-models, namely: HBV-only and TB-only sub-models are considered first of all. Unlike the HBV-only sub-model, which has a globally-asymptotically stable disease-free equilibrium whenever the associated reproduction number is less than unity, the TBonly sub-model undergoes the phenomenon of backward bifurcation, where a stable disease-free equilibrium co-exists with a stable endemic equilibrium, for a certain range of the associated reproduction number less than unity. Thus, for TB, the classical requirement of having the associated reproduction number to be less than unity, although necessary, is not sufficient for its elimination. It is also shown, that the full HBV-TB co-infection model undergoes a backward bifurcation phenomenon. Through simulations, we mainly find that i) the two diseases will co-exist whenever their partial reproductive numbers exceed unity; (ii) the increased progression rate due to exogenous reinfection from latent to active TB in co-infected individuals may play a significant role in the rising prevalence of $\mathrm{TB}$; and (iii) the increased progression rates from acute stage to chronic stage of HBV infection have increased the prevalence levels of HBV and TB prevalences.
\end{abstract}

Key words: nonlinear dynamical systems, epidemiological models, tuberculosis, hepatitis B, stability

AMS subject classification: 34A34, 34D23, 34D40, 92D30

*Corresponding author. E-mail: sbowong@gmail.com 


\section{Introduction}

Of the seven biggest killers worldwide, TB, malaria, hepatitis, and, in particular, HIV/AIDS continue to surge, with HIV/AIDS and TB likely to account for the overwhelming majority of deaths from infectious diseases in developing countries by 2020 [1-20]. Sub-Saharan Africa accounting for nearly half of infectious disease deaths globally will remain the most vulnerable region. The death rates for many diseases, including HIV/AIDS, tuberculosis, malaria and hepatitis B, exceed those in all other regions. Sub-Saharan Africa's health care capacity, the poorest in the world, will continue to lag [4,5]. Tuberculosis is an old disease whose world-wide prevalence had been diminishing even before vaccination and prophylaxis strategies were firstly accomplished. Its recent return in developing countries, mainly in Southeast Asia, have attracted renewed interest in it. The current world estimate of prevalence is about $33 \%$ while the number of deaths per year that it is causing reaches more than 3 million people [2]. Depending of the kind and the intensity of immune response that the host immune system performs after initial infection with $\mathrm{M}$. tuberculosis bacillus, the individual can suffer latent infection, in which the bacteria are under a growth-arrest regime and the individual neither suffer any clinical symptom nor becomes infectious or active infection, where the host suffers clinical symptoms and can transmit the pathogen by air. Latently infected individuals can, generally after an immune-depression episode, reach the active phase. Estimating the probability of developing direct active infection after a contact, or alternatively, the lifetime's risk for a latent infected individual to evolve into the active phase, are not easy tasks. However, it is generally accepted that only $5-10 \%$ of the infections directly produce active TB, while the ranges concerning the estimation of typical "half-life" of latent state rounds about 500 years $[4,9]$.

On the other hand, Hepatitis B is a disease caused by the hepatitis B virus (HBV) which infects the liver of hominoidae, including humans, and causes an inflammation called hepatitis. Originally known as "serum hepatitis", [3,10] the disease has caused epidemics in parts of Asia and Africa [10-20]. It can cause inflammation (swelling) of the liver, and sometimes significant liver damage. Many people do not even realize that they have been infected with that virus, because the typical flu-like symptoms may not develop immediately, or even at all. You can become infected with hepatitis B if you are not immune (resistant) to the virus and have been exposed to the blood or body fluids of an infected person. A vaccine is available to protect against hepatitis B. The hepatitis $B$ virus is present in body fluids such as blood, saliva, semen and vaginal fluid. It can be passed from person to person through unprotected sex (without using a condom) or by sharing needles to inject drugs, for example. Hepatitis B is 100 times more infectious than HIV. Infected mothers can also pass the virus to their baby during childbirth, often without knowing that they are infected. The incubation period (the time it takes from coming into contact with the virus to developing infection) is between one and six months. About a third of the world's population, more than 2 billion people, have been infected with the hepatitis B virus [3]. This includes 350 million chronic carriers of the virus. In developing countries, it is estimated that 5 to $15 \%$ of persons are chronic carriers, whereas only about $1 \%$ of the population is chronically infected in North America and Western Europe [3,10].

It is important to emphasize that the occurrence of tuberculosis (TB), human immunodeficiency 
virus (HIV), and viral hepatitis infections in the same patient poses unique clinical and public health challenges, because medications to treat TB and HIV are hepatotoxic [21]. In developing countries, the scale up of HIV treatment takes place within overlapping epidemics of TB and HBV, particularly in populations where injection drug use and poverty are widespread. The presence of all three infections creates conditions for a perfect storm in medical management, as treatments for TB and HIV each affect the liver already weakened by HBV. In the southwest provinces and some areas of central China, and sub-Saharan Africa, for example, many patients present with all three infections, leading to unique challenges in their medical care [22]. Tuberculosis and hepatitis B are the two common co-infections in patients infected with HIV [21-27]. TB remains an important public health problem in the world that has been exacerbated by the HIV epidemic, resulting in increased morbidity and mortality worldwide. Successful treatment of tuberculosis patients with viral hepatitis and HIV co-infection is a challenging task.

The symbiotic relationship between HBV and TB is a double blow to developing countries and in particular sub-Saharan Africa regions because of the high prevalence of TB and incidence of HBV. HBV and TB exhibit a unique symbiosis, despite biological differences. Therefore, there is a need for a strong qualitative assessment of the population-level implication of the consequences due to the interaction of these two diseases. Tuberculosis and HBV have become a major public health problem and show a danger mark even for the future generations, especially in Asian and sub-Saharan Africa. The main interest in studying HBV and TB model is to understand the long and short term behavior of the dynamics of both diseases and to predict whether the diseases will die out or will persist. A brief survey on previous studies provides the context of this paper.

Blal, et al. [21] studied the prevalence of hepatitis B virus infection among tuberculosis patients with and without HIV in Rio de Janeiro (Brazil). They showed that hepatitis B infection is highly prevalent in tuberculosis patients in Brazil and they suggested that a vaccination program for the general population should be considered in order to prevent further hepatitis B infections. In [22], the authors studied the risk factors and algorithms to identify hepatitis C, hepatitis B, and HIV among Georgian tuberculosis patients. They recruited 300 individuals at in-patient tuberculosis hospitals in three cities in Georgia, administered a behavioral questionnaire, and tested for antibody to HIV, hepatitis $\mathrm{C}(\mathrm{HCV})$, hepatitis B core antigen (anti-HBc), and the hepatitis B surface antigen (HBsAg). They found that HIV infection was rare but HBV and HCV infections were common among Georgian tuberculosis patients. High and increasing prevalence of HBV has been reported among both tuberculosis patients and blood donors in Georgia between 1998 and 2001. But this study was the first to indicate that HBV is a significant problem among patients with tuberculosis in Georgia. Bellamy et al. [23] analyzed the co-infection of tuberculosis and chronic hepatitis B virus infection in Africans. They found that persons with VDR genotype tt may be resistant to TB and persistence of HBV infection.

The study of infectious disease co-epidemics is critical for understanding how the diseases are related, and how prevention and treatment efforts can be most effective. Mathematical models can provide insight into the complicated infection dynamics, and into effective control measures. Most mathematical epidemic models evaluate a single disease [28,29], although a growing number of studies have considered co-epidemics. Mathematical studies of co-infection models are not very common. There are a few co-infection models (cf. [30-37]) in the literature. Nevertheless, 
Naresh, et al. [31] developed a nonlinear mathematical model for TB/HIV co-infection. Bacaer, et al. [33] developed a mathematical model for the joint dynamics of HIV and TB using numerical simulations to estimate parameters and predict the future transmission of TB in a South Africa township. Sharomi et al. [34] analyzed a mathematical model of the transmission dynamics of HIV/TB co-infection in the presence of treatment, while Mukandavire et al. [35] study a model for HIV-malaria co-infection. In [36], Expeditho, et al. presented an mathematical analysis of malaria and tuberculosis co-dynamics. Roeger, et al. [37] studied HIV/TB co-infection using a mathematical model which does not preclude the possibility of joint infections.

In this paper, we formulate and analyze a realistic mathematical model for HBV-TB co-infection, which incorporates the key epidemiological and biological features of each of the two diseases. The main contribution of this study is to carry out a detailed qualitative analysis of the resulting model. It is our view that this study represents the very first modelling work that provides an in-depth analysis of the qualitative dynamics of HBV-TB co-infection

The rest of this paper is organized as follows. The model is formulated in Section 2. The sub-models for HBV and TB are presented and analyzed in Sections 3. The analysis of the full HBV-TB co-infection model is carried out in Section 4. Numerical results are presented to illustrate the analytical results. Finally, section 5 contains concluding remarks.

\section{Modeling of tuberculosis and HBV co-dynamics}

\subsection{Model construction}

The formulation of this co-infection closely follows the epidemiological dynamics of the two diseases.

\subsubsection{Basic framework}

The model sub-divides the total population at time $t$ denoted by $N(t)$, into various mutuallyexclusive compartments depending on their disease status: Susceptible individuals to both diseases $(S(t))$, immune individuals following vaccination to HBV disease $(V(t))$, individuals with latent $\mathrm{TB}, E_{T}(t)$, individuals with active TB $\left(I_{T}(t)\right)$, individuals with $\mathrm{HBV}$ acute infection $\left(I_{H}(t)\right)$, $\mathrm{HBV}$ chronic carriers $\left(C_{H}(t)\right)$, HBV recovered individuals with protective immunity $\left(R_{H}(t)\right)$ duallyinfected individuals with $\mathrm{HBV}$ acute infection, in the TB latent stage $\left(E_{T I}(t)\right)$, dually-infected individuals with HBV acute infection, in the TB active stage $\left(I_{T I}(t)\right)$, dually-infected individuals with HBV chronic carrier, in the TB latent stage $\left(E_{T C}(t)\right)$, dually-infected individuals with HBV chronic carrier, in the TB active stage $\left(I_{T C}(t)\right)$, HBV recovered individuals with protective immunity in the TB latent stage $\left(E_{T R}(t)\right)$ and HBV recovered individuals with protective immunity in the TB active stage $\left(I_{T R}(t)\right)$. 
The total population is

$$
\begin{aligned}
N(t) & =S(t)+V(t)+E_{T}(t)+I_{T}(t)+I_{H}(t)+C_{H}(t)+R_{H}(t)+E_{T I}(t)+I_{T I}(t) \\
& +E_{T C}(t)+I_{T C}(t)+E_{T R}(t)+I_{T R}(t) .
\end{aligned}
$$

The compartmental diagram in Fig. 1 illustrates the flow of individuals as they face the possibility of acquiring specific-disease infections or even co-infections.

The model is based on the following assumptions. The mixing between individuals is homogeneous. The population of individuals infected with active-TB is only generated from latent TB class following infection. Latently infected individuals are assumed to acquire some immunity as a result of infection, which reduces the risk of subsequent infection but does not fully prevent it. HBV carrier mothers can pass the virus to their baby during the childbirth. The vaccine-induced immunity acquired by vaccinated individuals is supposed to wane and the way for vaccinated individuals to acquire HBV infection is through the waning of vaccine-induced protection, and individuals therefore enter the HBV acute infection class.

All HBV infected individuals (those in the $I_{H}, C_{H}$ and $R_{H}$ classes) are susceptible to TB infection and TB infected individuals (those in the $E_{T}$ and $I_{T}$ classes) are susceptible to HBV infection. Dually infected individuals with TB in the acute and chronic stage of HBV infection (those in the $E_{T I}, I_{T I}, E_{T C}$ and $I_{T C}$ classes) are assumed to transmit $\mathrm{HBV}$ at the same rate $\lambda_{H}$ as those with only HBV infection ( $I_{H}$ or $C_{H}$ class). Individuals in the $I_{T I}, I_{T C}$ and $I_{T R}$ classes can transmit TB at the same rate $\lambda_{T}$ as individuals with active TB alone ( $I_{T}$ class). Susceptible and $\mathrm{HBV}$ vaccinated individuals acquire TB infection following effective contact with TB-infected individuals in the infectious stage (at rate $\lambda_{T}$ ). Also, susceptible who have never been successfully vaccinated, or who have been vaccinated but the protective effect of vaccination worn off acquire infection with HBV following effective contact with individuals in the acute and chronic carriers stage of HBV infection (at rate $\lambda_{H}$ ).

The force of infection associated with the HBV infection, denoted by $\lambda_{H}$, is given by

$$
\lambda_{H}=\beta_{H} \frac{I_{H}+\eta_{1}\left(E_{T I}+\eta_{1 c} I_{T I}\right)+\eta\left(C_{H}+\eta_{2}\left(E_{T C}+\eta_{2 c} I_{T C}\right)\right)}{N},
$$

where $\beta_{H}$ is the effective contact rate for HBV infection (contact sufficient to result in HBV infection), the modification parameter $\eta_{1} \geq 1$ accounts for the relative infectiousness of individuals with HBV acute infection in the TB latent stage $\left(E_{T I}\right)$ or displaying active TB $\left(I_{T I}\right)$ in comparison to those with HBV infection alone but with acute infection $\left(I_{H}\right)$. In other words, it is assumed that $\mathrm{HBV}$-infected individuals (with acute infection) who are also infected with TB are more infectious than HBV-infected individuals with acute infection and no TB infection (similar comparisons are made for HBV chronic carriers alone in relation to those with HBV chronic carriers and TB infection). Further, the parameter $\eta_{1 c}$ (with $\eta_{1 c} \geq \eta_{1} \geq 1$ ) captures the fact that dually-infected individuals with HBV acute infection, but displaying active TB $\left(I_{T I}\right)$, are more infectious than the corresponding dually-infected individuals with HBV acute infection in the TB latent stage $\left(E_{T I}\right)$. The parameter $\eta$ models the reduced transmission rate of individuals in the chronic stage of HBV infection with respect to the infectiousness of individuals in the acute infection of HBV 


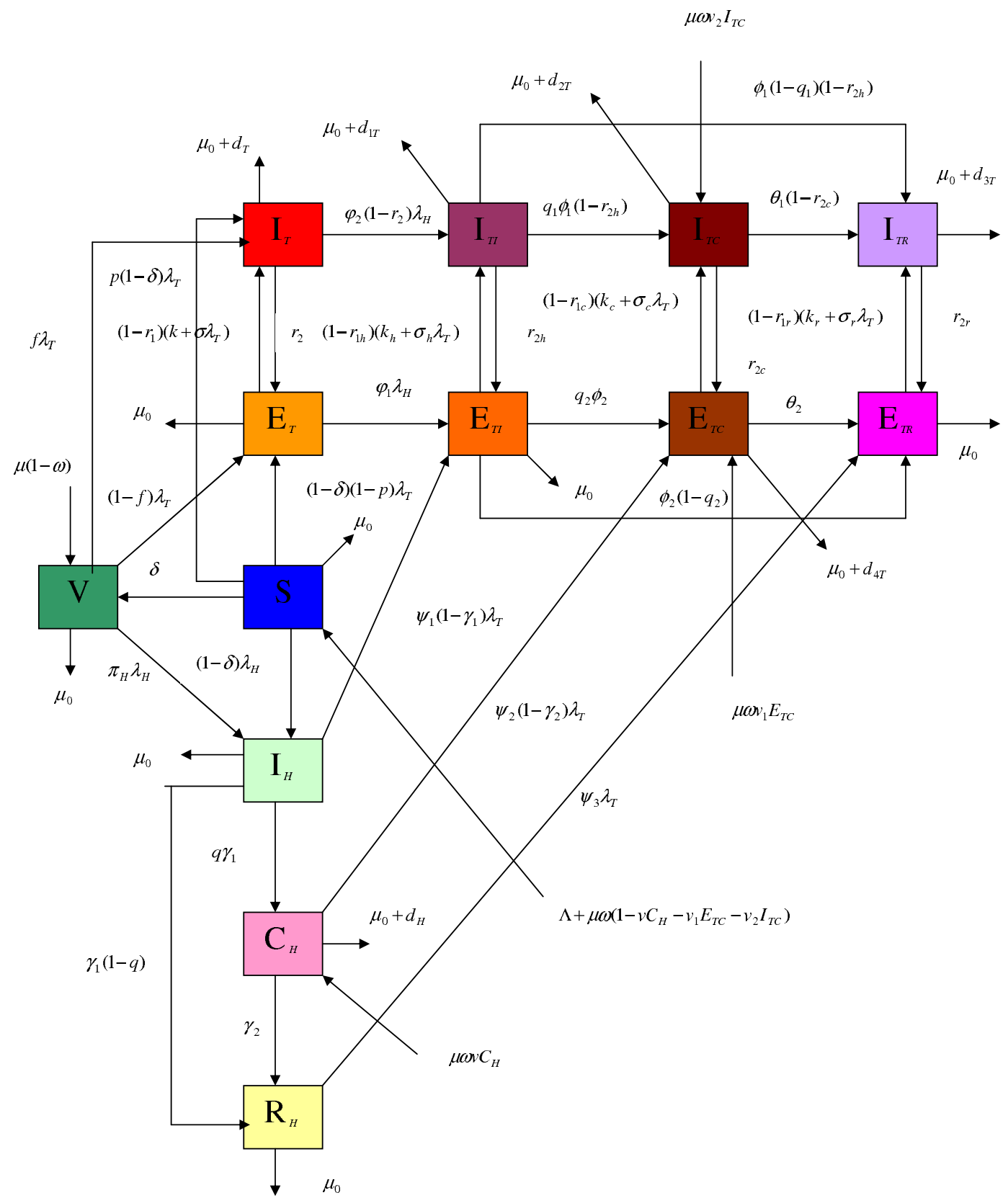

Figure 1: Flowchart of the transmission dynamics of the co-infection TB/HBV.

infection alone. This is due to the fact that individuals in the acute stage of HBV infection have higher viral load compared to individuals in the chronic stage of HBV infection(this is owing to the aforementioned correlation between HBV viral load and infectiousness). Also, $\eta_{2}$ models the relative infectiousness of dually-infected individuals with chronic infection of $\mathrm{HBV}$ in the latent stage of TB $\left(E_{T C}\right)$ or displaying active TB $\left(I_{T C}\right)$ in comparison to those with HBV chronic infec- 
tion alone $\left(C_{H}\right)$. Finally, $\eta_{2 c}$ (with $\eta_{2 c} \geq \eta_{2}$ ) is the modification parameter accounting the fact that dually-infected individuals with HBV chronic infection, but displaying active TB $\left(I_{T C}\right)$, are more infectious than the corresponding dually-infected individuals with HBV chronic infection in the TB latent stage $\left(E_{T C}\right)$.

Similarly, susceptible and HBV vaccinated individuals acquire TB infection following contact with people in the active stage of $\mathrm{TB}$ (that is, those in $I_{T}, I_{T I}, I_{T C}$ and $I_{T R}$ classes) at rate $\lambda_{T}$ defined as follows:

$$
\lambda_{T}=\beta_{T} \frac{I_{T}+\varepsilon\left(I_{T R}+\varepsilon_{t} I_{T I}+\varepsilon_{c} I_{T C}\right)}{N},
$$

where $\beta_{T}$ is the contact rate associated with the TB infection. The modification parameter $\varepsilon>1$ accounts for the relative risk of infectiousness of dually-infected individuals in the active-TB classes $\left(I_{T I}, I_{T C}\right.$ and $\left.I_{T R}\right)$ in comparison to those in the only active TB class $\left(I_{T}\right)$. Further, the modification parameter $\varepsilon_{t}$ (with $\varepsilon_{t}>\varepsilon>1$ ) accounting to the fact that dually-infected individuals with HBV acute infection, but displaying that active TB $\left(I_{T I}\right)$ are more infectious than the corresponding HBV recovered individuals in the TB active stage $\left(I_{T R}\right)$. Finally, $\varepsilon_{c}$ (with $\varepsilon_{c} \geq \varepsilon_{t}>1$ ) accounts for the assumed increase in infectiousness for dually-infected individuals in the chronic stage of HBV infection, but displaying active TB $\left(I_{T C}\right)$, compared to dually-infected individuals with HBV acute infection in the TB active stage $\left(I_{T I}\right)$.

We define by $\omega$ the proportion of births without successful vaccination. Children born by carrier mothers (in the $C_{H}, E_{T C}$ and $I_{T C}$ classes) who are unsuccessfully immunized by vaccination or have not been vaccinated can be infected by carrier mothers and will enter the $C_{H}, E_{T C}$ and $I_{T C}$ classes at rates $\mu \omega, \mu \omega v_{1}$ and $\mu \omega v_{2}$, respectively.

The susceptible class is generated by the recruitment of individuals into the population from immigration and children born by carrier women without successful vaccination who have not been infected by the HBV virus at the rates, $\Lambda$ and $\mu \omega\left(1-v C_{H}-v_{1} E_{T C}-v_{2} I_{T C}\right)$, respectively. The HBV vaccinated population increases by births who are successful immunized, and are protected from perinatal and postnatal infection at rate $\mu(1-\omega)$. Every susceptible vaccinated neonate enters the vaccinated class $V$ at rate $\delta$, representing the assumption that vaccination prevents perinatal transmission from infectious women. We assume that this protection is not lifelong. Further, natural death rate occurs in all compartments at the rate $\mu_{0}$.

Individuals with latent TB who have been infected with Mycobacterium tuberculosis but have no clinical symptoms/illness and are non-infectious. Fraction $p$ of newly infected susceptible individuals is assumed to undergo a fast progression directly to the infectious class $I_{T}$, while the remainder is latently infected and enter the latent class $E_{T}$. Similarly, a fraction $f$ of HBV vaccinated individuals who acquire TB infection move to the TB latent class $\left(E_{T}\right)$, while the remaining fraction, $1-f$, enters the TB active class $\left(I_{T}\right)$. Once latently infected with M. Tuberculosis, an individual will remain so for life unless reactivation occurs. To account for treatment, we define by $r_{1} E_{T}$ the fraction of latently infected individuals receiving effective chemoprophylaxis, and $r_{2}$ as the rate of effective per capita therapy of individuals in the active TB alone. Chemoprophylaxis of latently infected individuals reduces their reactivation at the rate $r_{1}$ and the initiation of therapeutics immediately removes individuals from active status $I_{T}$ and places them into a latent state $E_{T}$ at the rate $r_{2}$. Due to endogenous reactivation, a fraction $1-r_{1}$ of latently infected individuals who 
did not received effective chemoprophylaxis become infectious with a rate $k$, and some become reinfected (exogenously) after effective contact with individuals in the active TB classes only at rate $\sigma \lambda_{T} . \sigma$ models the factor reducing the risk of infection as a result of acquiring immunity for latently infected individuals. Individuals with active-TB alone suffer a disease-induced death rate $d_{T}$.

The population of individuals infected with TB only (in the $E_{T}$ or $I_{T}$ class) is also reduced following acquisition of HBV-infection, which can result following effective contacts with individuals in the HBV acute and chronic infection stage (in the $I_{H}, C_{H}, E_{T I}, E_{T C}, I_{T I}$ and $I_{T C}$ classes). Individuals exposed to TB can be infected with HBV (at a rate $\lambda_{H}$ ). That is, individuals in the $E_{T}$ class are moved into the $E_{T I}$ class upon acquiring HBV infection at rate $\varphi_{1} \lambda_{H}$ where $\varphi_{1}$ accounts for the assumed increase in the probability of acquiring HBV infection for TB latently infected individuals only. Furthermore, individuals in the active stage of TB infection alone can acquire HBV infection, at rate $\varphi_{2}\left(1-r_{2}\right) \lambda_{H}$ where $\varphi_{2}$ (with $\varphi_{2}>\varphi_{1}$ ) models the probability of acquiring $\mathrm{HBV}$ infection for individuals in the active stage of TB infection alone. Individuals in the $E_{T I}$ class progress to the $E_{T C}$ and $E_{T R}$ classes at rates $q_{2} \phi_{2}$ and $\phi_{2}\left(1-q_{2}\right)$, respectively, where $q_{2}$ is the average probability that an individual in the $E_{T I}$ class fails to clear an acute infection and develops a carrier state $\left(E_{T C}\right)$. Similarly, individuals in the $I_{T I}$ class progress to the $I_{T C}$ and $I_{T R}$ classes at rates $q_{1} \phi_{1}$ and $\phi_{1}\left(1-q_{1}\right)$, respectively, where $q_{1}$ is the average probability that an individual in the $I_{T I}$ class fails to clear an acute infection and develops a carrier state $\left(I_{T C}\right)$. Dually infected individuals with HBV acute and chronic infection, and HBV recovered individuals in the TB latent stage are assumed to receive effective chemoprophylaxis at rates $r_{1 h}, r_{1 c}$ and $r_{1 r}$ (with $\left.r_{1 r}>r_{1 h}>r_{1 c}\right)$, respectively.

Dually infected individuals with HBV acute and chronic infection, and HBV recovered individuals in the TB latent stage (in the $E_{T I}, E_{T C}$ and $E_{T R}$ classes) who did not receive effective chemoprophylaxis can develop an active TB and will move to the $I_{T I}, I_{T C}$ and $I_{T R}$ classes at rates $k_{h}\left(1-r_{1 h}\right), k_{c}\left(1-r_{1 c}\right)$ and $k_{r}\left(1-r_{1 r}\right)$ for endogenous reactivation and $\sigma_{h} \lambda_{T}, \sigma_{c} \lambda_{T}$ and $\sigma_{r} \lambda_{T}$ for exogenous re-infection, respectively. $\sigma_{h}, \sigma_{c}$ and $\sigma_{r}$ are respectively, the factors reducing the risk of infection as a result of acquiring immunity for individuals in the $E_{T I}, E_{T C}$ and $E_{T R}$ classes. The initiation of therapeutics immediately removes individuals from active status $I_{T I}, I_{T C}$ and $I_{T R}$ and places them into latent states $E_{T I}, E_{T C}$ and $E_{T R}$ at rates $r_{2 h}, r_{2 c}$ and $r_{2 r}$ (with $r_{2 r}>r_{2 h}>r_{2 c}$ ), respectively. Furthermore, individuals in the $I_{T I}$ and $I_{T R}$ classes die due to TB at rates $d_{1 T}$ and $d_{3 T}$ (with $d_{1 T}>d_{3 T}$ ), respectively, while, individuals in the $E_{T C}$ class die due to HBV at rate $d_{4 T}$. In addition, individuals in the $I_{T C}$ class suffer death due to TB and HBV at rate $d_{2 T}$ (with $d_{2 T}>d_{4 T}>d_{H}$ ). We point out that since the chemoprophylaxis of latently infected individuals only reduces their reactivation to active $\mathrm{TB}$, chemoprophylaxis of dually-infected individuals with latent TB and $\mathrm{HBV}$ infection (those in the $E_{T}, E_{T I}$ and $E_{T C}$ classes) have no effect on the progression of the HBV infection. This is why there, the terms $1-r_{1}, 1-r_{1 h}$ and $1-r_{1 c}$ which are associated to the chemoprophylaxis of individuals in the $E_{T}, E_{T I}$ and $E_{T C}$ classes do not exist in the expressions of the transfer rates from $E_{T}$ to $E_{T I}$, from $E_{T I}$ to $E_{T C}$ and from $E_{T C}$ to $E_{T R}$, respectively. 
Table 1: Description of the variables of the model

\begin{tabular}{cc}
\hline \hline Variable & Description \\
\hline$S(t)$ & Susceptible individuals \\
$V(t)$ & TB infected individuals in the TB latent stage \\
$E_{T}(t)$ & TB-infected individuals in the active stage of TB \\
$I_{T}(t)$ & Dually-infected individuals with HBV acute infection \\
$E_{T I}(t)$ & in the TB latent stage \\
& displaying active TB \\
$I_{T I}(t)$ & Dually-infected individuals with HBV acute infection that \\
& in the TB latent stage chronic carrier \\
$E_{T C}(t)$ & Dually-infected individuals with HBV \\
& displaying active TB \\
$I_{T C}(t)$ & Dually-infected individuals with HBV chronic carrier that \\
& in the TB latent stage \\
$E_{T R}(t)$ & HBV recovered individuals with protective immunity \\
& displaying active TB \\
$I_{T R}(t)$ & HBV recovered individuals with protective immunity that \\
& HBV acute infection individuals \\
$I_{H}(t)$ & HBV chronic carriers \\
$C_{H}(t)$ & HBV recovered individuals with protective immunity \\
$R_{H}(t)$ &
\end{tabular}

Susceptible individuals whose HBV vaccination was successful enter in the HBV vaccinated class $V$ at rate $\delta$, while the remaining who can be infected with $\mathrm{HBV}$ and will move to the $I_{H}$ class with the force of infection $(1-\delta) \lambda_{H}$. The vaccine-induced immunity acquired by vaccinated individuals is supposed to wane at rate $\pi_{H}$ and the way to vaccinated individuals to acquire HBV infection is through the waning of vaccine-induced protection, and individuals therefore enter the HBV acute infection class $I_{H}$. Thus, HBV vaccinated individuals can experience break-through infection (due to incomplete protection provided by vaccine) and can become infected with HBV at rate $\pi_{H} \lambda_{H}$. We define by $q$ the average probability that an individual in the HBV acute class $I_{H}$ fails to clear an acute infection but develops a chronic state $\left(C_{H}\right)$. Then, individuals in the $I_{H}$ class progress to the $C_{H}$ class at rate $q \gamma_{1}$, while the remainders enter the $R_{H}$ class at rate $\gamma_{1}(1-q)$. Further, individuals in the $C_{H}$ class progress to the HBV recovered class $\left(R_{H}\right)$, at rate $\gamma_{2}$. Individuals in the $I_{H}, C_{H}$ and $R_{H}$ classes are also reduced due to the TB infection (following effective contacts with individuals in the $I_{T}, I_{T I}, I_{T C}$ and $I_{T R}$ classes). The parameters $\psi_{1}, \psi_{2}$ and $\psi_{3}$ (with $\psi_{3}<\psi_{1}<\psi_{2}$ ) account for the assumed increase in probability of acquiring TB infection for HBV-infected individuals alone. The parameters $\psi_{1}, \psi_{2}$ and $\psi_{3}$ are associated, respectively, with those with acute infection $\left(I_{H}\right)$, carrier state $\left(C_{H}\right)$ and recovered state $\left(R_{H}\right)$. It is assumed that individuals with HBV infection are more prone to TB infection than all susceptible individuals. Further, those with HBV chronic state acquire TB infection at a higher rate than those in the acute stage of HBV infection which also acquire TB infection at a higher rate than recovered individuals owing to the weaker immune status of the former. Once infected by Mycobacterium 
tuberculosis, individuals in the $I_{H}, C_{H}$ and $R_{H}$ classes move to the $E_{T I}, E_{T C}$ and $E_{T R}$ classes at rates $\psi_{1}\left(1-\gamma_{1}\right) \lambda_{T}, \psi_{2}\left(1-\gamma_{2}\right) \lambda_{T}$ and $\psi_{3} \lambda_{T}$, respectively. Carriers are prone to the background death rate that applies to the whole community but have an additional death rate $d_{H}$ because of their hepatitis B carrier status.

The model variables and parameters are further described in Tables 1 and 2 for elucidation.

Table 2: Description of parameters of the model

\begin{tabular}{|c|c|}
\hline Parameters & Description \\
\hline$\Lambda$ & Recruitment rate of susceptible individuals into the population \\
\hline$\beta_{T}$ & Transmission rate of TB \\
\hline$\beta_{H}$ & Transmission rate of $\mathrm{HBV}$ \\
\hline$\eta_{1}, \eta_{1 c}$ & $\begin{array}{l}\text { Factors taking into account the infectivity of } \\
\text { individuals in the HBV acute infection stage }\end{array}$ \\
\hline$\eta, \eta_{2}, \eta_{2 c}$ & Infectiousness of individuals in the chronic stage of $\mathrm{HBV}$ infection \\
\hline$\varepsilon, \varepsilon_{t}, \varepsilon_{c}$ & Factors taking into account the infectivity of active TB individuals \\
\hline$\pi_{H}$ & Rate of waning vaccine-induced immunity \\
\hline$p$ & Fraction of newly-infected susceptible individuals with latent TB \\
\hline$f$ & Fraction of newly-infected HBV vaccinated individuals with latent TB \\
\hline$\varphi_{1}$ & $\begin{array}{l}\text { Probability of acquiring HBV infection of } \\
\text { individuals in the } E_{T} \text { class }\end{array}$ \\
\hline$\varphi_{2}$ & Probability of acquiring HBV infection of individuals in the $I_{T}$ class \\
\hline$\psi_{1}$ & Probability of acquiring TB of individuals in the $I_{H}$ class \\
\hline$\psi_{2}$ & Probability of acquiring TB of individuals in the $C_{H}$ class \\
\hline$\psi_{3}$ & Probability of acquiring TB of individuals in the $R_{H}$ class \\
\hline$k$ & Progression rate from the $E_{T}$ class to the $I_{T}$ class \\
\hline$k_{h}$ & Progression rate from the $E_{T I}$ class to the $I_{T I}$ class \\
\hline$k_{c}$ & Progression rate from the $E_{T C}$ class to the $I_{T C}$ class \\
\hline$k_{r}$ & Progression rate from the $E_{T R}$ class to the $I_{T R}$ class \\
\hline$\sigma, \sigma_{h}, \sigma_{c}, \sigma_{r}$ & Re-infection parameters in the $E_{T}, E_{T I}, E_{T C}$ and $E_{T R}$ classes \\
\hline$\mu_{0}$ & Natural death rate of people \\
\hline$\mu$ & Birth rate in the population \\
\hline$\omega$ & Proportion of births without successful vaccination \\
\hline$v, v_{1}, v_{2}$ & $\begin{array}{c}\text { Proportions of perinatally infected born by carriers mothers } \\
\text { in the } C_{H}, E_{T C} \text { and } I_{T C} \text { classes }\end{array}$ \\
\hline$\delta$ & Vaccination rate \\
\hline$\gamma_{1}, \phi_{1}, \phi_{2}$ & Rate moving from acute to carrier \\
\hline$q, q_{1}, q_{2}$ & $\begin{array}{l}\text { Average probability that an individual in the } \mathrm{HBV} \text { acute infection fail to clear } \\
\text { an acute state and develops a carrier state }\end{array}$ \\
\hline $\begin{array}{c}\gamma_{2}, \theta_{1}, \theta_{2} \\
d_{H}\end{array}$ & $\begin{array}{l}\text { Rate moving from carrier classes to recovered classes } \\
\mathrm{HBV} \text { induced death rate of individuals in the } \mathrm{HBV} \text { chronic carriers class } C_{H}\end{array}$ \\
\hline
\end{tabular}


$d_{T} \quad$ TB induced death rate of individuals in the TB active class $I_{T}$

$d_{1 T} \quad$ TB induced death rate of individuals in the $I_{T I}$ class

$d_{2 T}$

$\mathrm{HBV}$ and TB induced death rate of individuals in the $I_{T C}$ class

$d_{3 T}$

TB induced death rate of individuals in the $I_{T R}$ class

$d_{4 T}$

$\mathrm{HBV}$ induced death rate of individuals in the $E_{T C}$ class

$r_{2}$

TB recovery rate of individuals in the $I_{T}$ class

$r_{2 h}$

TB recovery rate of individuals in the $I_{T I}$ class

$r_{2 c}$

TB recovery rate of individuals in the $I_{T C}$ class

TB recovery rate of individuals in the $I_{T R}$ class

$r_{1} \quad$ Rate of TB effective chemoprophylaxis of individuals in the $E_{T}$ class

$r_{1 h} \quad$ Rate of TB effective chemoprophylaxis of individuals in the $E_{T I}$ class

$r_{1 c}$ Rate of TB effective chemoprophylaxis of individuals in the $E_{T C}$ class

$r_{1 r} \quad$ Rate of TB effective chemoprophylaxis of individuals in the $E_{T R}$ class

\subsubsection{The model}

Putting the above formulations and assumptions together gives the following system of differential equations:

$$
\left\{\begin{aligned}
\dot{S} & =\Lambda+\mu \omega\left(1-v C_{H}-v_{1} E_{T C}-v_{2} I_{T C}\right)-(1-\delta)\left(\lambda_{T}+\lambda_{H}\right) S-\left(\mu_{0}+\delta\right) S \\
\dot{V} & =\mu(1-\omega)+\delta S-\left(\lambda_{T}+\pi_{H} \lambda_{H}\right) V-\mu_{0} V \\
\dot{I}_{H} & =\left[\pi_{H} V+(1-\delta) S\right] \lambda_{H}-\psi_{1}\left(1-\gamma_{1}\right) \lambda_{T} I_{H}-\left(\mu_{0}+\gamma_{1}\right) I_{H} \\
\dot{C}_{H} & =\mu \omega v C_{H}+q \gamma_{1} I_{H}-\psi_{2}\left(1-\gamma_{2}\right) \lambda_{T} C_{H}-\left(\mu_{0}+d_{H}+\gamma_{2}\right) C_{H} \\
\dot{R}_{H} & =\gamma_{1}(1-q) I_{H}+\gamma_{2} C_{H}-\psi_{3} \lambda_{T} R_{H}-\mu_{0} R_{H} \\
\dot{E}_{T} & =[(1-f) V+(1-\delta)(1-p) S] \lambda_{T}+r_{2} I_{T}-\left(1-r_{1}\right)\left(k+\sigma \lambda_{T}\right) E_{T} \\
& -\varphi_{1} \lambda_{H} E_{T}-\mu_{0} E_{T}, \\
\dot{I}_{T} & =[f V+p(1-\delta) S] \lambda_{T}+\left(1-r_{1}\right)\left(k+\sigma \lambda_{T}\right) E_{T}-\varphi_{2}\left(1-r_{2}\right) \lambda_{H} I_{T} \\
& -\left(\mu_{0}+d_{T}+r_{2}\right) I_{T}, \\
\dot{E}_{T I} & =\varphi_{1} \lambda_{H} E_{T}+\psi_{1}\left(1-\gamma_{1}\right) \lambda_{T} I_{H}+r_{2 h} I_{T I}-\sigma_{h}\left(1-r_{1 h}\right) \lambda_{T} E_{T I} \\
& -\left[\mu_{0}+k_{h}\left(1-r_{1 h}\right)+\phi_{2}\right] E_{T I}
\end{aligned}\right.
$$




$$
\left\{\begin{aligned}
\dot{I}_{T I} & =\varphi_{2}\left(1-r_{2}\right) \lambda_{H} I_{T}+\left(1-r_{1 h}\right)\left(k_{h}+\sigma_{h} \lambda_{T}\right) E_{T I} \\
& -\left[\mu_{0}+d_{1 T}+r_{2 h}+\phi_{1}\left(1-r_{2 h}\right)\right] I_{T I}, \\
\dot{E}_{T C} & =\mu \omega v_{1} E_{T C}+q_{2} \phi_{2} E_{T I}+\psi_{2}\left(1-\gamma_{2}\right) \lambda_{T} C_{H}+r_{2 c} I_{T C} \\
& -\sigma_{c}\left(1-r_{1 c}\right) \lambda_{T} E_{T C}-\left[\mu_{0}+d_{4 T}+\theta_{2}+k_{c}\left(1-r_{1 c}\right)\right] E_{T C}, \\
\dot{I}_{T C} & =\mu \omega v_{2} I_{T C}+q_{1} \phi_{1}\left(1-r_{2 h}\right) I_{T I}+\left(1-r_{1 c}\right)\left(k_{c}+\sigma_{c} \lambda_{T}\right) E_{T C} \\
& -\left[\mu_{0}+d_{2 T}+r_{2 c}+\theta_{1}\left(1-r_{2 c}\right)\right] I_{T C}, \\
\dot{E}_{T R} & =\psi_{3} \lambda_{T} R_{H}+\theta_{2} E_{T C}+\phi_{2}\left(1-q_{2}\right) E_{T I}+r_{2 r} I_{T R} \\
& -\left(1-r_{1 r}\right) \sigma_{r} \lambda_{T} E_{T R}-\left[\mu_{0}+k_{r}\left(1-r_{1 r}\right)\right] E_{T R}, \\
\dot{I}_{T R} & =\theta_{1}\left(1-r_{2 c}\right) I_{T C}+\phi_{1}\left(1-q_{1}\right)\left(1-r_{2 h}\right) I_{T I}+\left(1-r_{1 r}\right)\left(k_{r}+\sigma_{r} \lambda_{T}\right) E_{T R} \\
& -\left(\mu_{0}+d_{3 T}+r_{2 r}\right) I_{T R} .
\end{aligned}\right.
$$

We assume that the population of new born carriers (born by carrier mothers) is less than the sum of the death of carriers and the population moving from carrier to immune state. In this case, we have $\mu \omega v<\mu_{0}+d_{H}+\gamma_{2}$. Otherwise, carriers would keep increasing rapidly as long as there is infection, i.e., $d C_{H} / d t>0$ for $C_{H} \neq 0$ or $I_{H} \neq 0$ and $t \geq 0$.

The parameter values used for numerical simulation are given in Table 3.

Table 3: Numerical values for the parameters of the model

\begin{tabular}{ccc}
\hline \hline Parameters & Estimated values/range & Reference \\
\hline$\Lambda$ & 500 per year & Assumed \\
$\beta_{T}$ & Variable & \\
$\beta_{H}$ & Variable & \\
$\pi_{H}$ & 0.1 per year & {$[12]$} \\
$\eta$ & 0.16 per year & {$[11]$} \\
$\eta_{1}, \eta_{1 c}, \eta_{2}, \eta_{2 c}$ & $0.6,1.1,1.2,1.4$ per year & Assumed \\
$\varepsilon, \varepsilon_{t}, \varepsilon_{c}$ & $1.1,1.3,1.5$ per year & Assumed \\
$p$ & 0.7 per year & Assumed \\
$f$ & 0.6 per year & Assumed
\end{tabular}




\begin{tabular}{ccc}
$\varphi_{1}, \varphi_{2}$ & $0.3,0.5$ per year & Assumed \\
$\psi_{1}, \psi_{2}, \psi_{3}$ & $0.4,0.6,0.1$ per year & Assumed \\
$k$ & 0.00013 per year & {$[40]$} \\
$k_{h}, k_{c}, k_{r}$ & $0.00015,0.00016,0.00014$ per year & Assumed \\
$\sigma, \sigma_{h}, \sigma_{c}, \sigma_{r}$ & $0.7,0.75,0.8,0.9$ per year & Assumed \\
$\mu_{0}$ & 0.019896 per year & {$[42]$} \\
$\mu$ & 0.0121 per year & {$[42]$} \\
$\omega$ & 0.1 per year & Assumed \\
$v$ & 0.11 per year & {$[11]$} \\
$v_{1}, v_{2}$ & $0.12,0.13$ per year & Assumed \\
$\delta$ & 0.7 per year & Assumed \\
$\gamma_{1}$ & 4 per year & {$[11]$} \\
$\phi_{1}, \phi_{2}$ & 2,3 per year & Assumed \\
$q$ & 0.0885 per year & {$[39]$} \\
$q_{1}, q_{2}$ & $0.9,0.95$ per year & Assumed \\
$\gamma_{2}$ & 0.025 per year & {$[11]$} \\
$\theta_{1}, \theta_{2}$ & $0.015,0.01$ per year & Assumed \\
$d_{H}$ & 0.002 per year & Assumed \\
$d_{T}$ & 0.0575 per year & {$[41]$} \\
$d_{1 T}, d_{2 T}, d_{3 T}, d_{4 T}$ & $0.06,0.08,0.05,0.07$ per year & Assumed \\
$r_{2}$ & 0.7311 per year & {$[41]$} \\
$r_{2 h}, r_{2 c}, r_{2 r}$ & $0.5,0.4,0.6$ per year & {$[41]$} \\
$r_{1}, r_{1 h}, r_{1 c}, r_{1 r}$ & $0,0,0,0$ per year & {$[38]$} \\
\hline \hline
\end{tabular}

\subsection{Positivity of solutions}

For the system (2.4) to be epidemiologically meaningful, it is important to prove that all its state variables are non-negative for all time. In other word, solutions of system (2.4) with positive initial data remain positive for all time $t>0$. This can be verified as follows. Suppose, for example, the variable $I_{H}$ becomes zero for some time $\bar{t}>0$, i.e., $I_{H}(\bar{t})=0$, while all other variables are positive. Then, from the $I_{H}$ equation we have $d I_{H}(\bar{t}) / d t>0$. Thus, $I_{H}(t) \geq 0$ for all $t>0$. Similarly, it can be shown that all variables remain nonnegative for all $t>0$.

\subsection{Invariant region}

The TB-HBV transmission model (2.4) will therefore be analyzed in a suitable feasible region, obtained as follows.

We first show that all feasible solutions are uniformly-bounded in a proper subset of $\Omega$. Let, $\left(S, V, I_{H}, C_{H}, R_{H}, E_{T}, I_{T}, E_{T I}, E_{T C}, E_{T R}, I_{T I}, I_{T C}, I_{T R}\right) \in \mathbb{R}_{>0}^{13}$ be any solution of the system with non-negative initial conditions. Adding all the equations in system (2.4) gives the following equation for $N$, the total population

$$
\dot{N}=\Lambda+\mu-\mu_{0} N-\left(d_{H} C_{H}+d_{T} I_{T}+d_{1 T} I_{T I}+d_{4 T} E_{T C}+d_{2 T} I_{T C}+d_{3 T} I_{T R}\right) .
$$


Then, one gets $\dot{N}<\Lambda+\mu-\mu_{0} N$. Thus, applying Birkhoff's and Rota's Theorem on differential inequality [43], as $t \rightarrow \infty$, one obtains $0 \leq N \leq \frac{\Lambda+\mu}{\mu_{0}}$. Therefore, all feasible solutions of components of system (2.4) enter the region

$$
\Omega=\left\{\left(S, V, I_{H}, C_{H}, R_{H}, E_{T}, I_{T}, E_{T I}, E_{T C}, E_{T R}, I_{T I}, I_{T C}, I_{T R}\right) \in \mathbb{R}_{\geq 0}^{13}, \quad N(t) \leq \frac{\Lambda+\mu}{\mu_{0}}\right\} .
$$

Thus, it follows from (2.5) that all possible solutions of system (2.4) will enter the region. Hence, the region $\Omega$, of biological interest, is positively-invariant under the flow induced by system (2.4). Further, it can be shown using the theory of permanence [44] that all solutions on the boundary of $\Omega$ eventually enter the interior of $\Omega$. Furthermore, in $\Omega$, the usual existence, uniqueness and continuation results hold for system (2.4). Hence, system (2.4) is well posed mathematically and epidemiologically and it is sufficient to consider the dynamics of the flow generated by the system (2.4) in $\Omega$.

Having established a biologically feasible region where the model is biologically and mathematically well-posed [45], the next step is to consider the dynamics of the two sub-models, namely; HBV-only and TB-only models. This would help to lay down the foundation for the qualitative analysis of the full model.

\section{Mathematical analysis of the HBV and TB sub-models}

\subsection{HBV-only sub-model}

Hepatitis B is a potentially life-threatening liver infection caused by the HBV and is a major global health problem and the most serious type of viral hepatitis [3]. It has caused epidemics in parts of Asia and Africa. Worldwide, about 2 billion people have been infected with the virus and about 360 million live with this chronic infection. Estimated 600,000 persons die each year due to the acute or chronic consequences of hepatitis B $[3,46]$.

The HBV-only model is obtained by setting $E_{T}=I_{T}=E_{T I}=I_{T I}=E_{T C}=I_{T C}=E_{T R}=$ $I_{T R}=0$. Thus, we have,

$$
\left\{\begin{aligned}
\dot{S} & =\Lambda+\mu \omega\left(1-v C_{H}\right)-(1-\delta) \lambda_{H} S-\left(\delta+\mu_{0}\right) S \\
\dot{V} & =\mu(1-\omega)+\delta S-\pi_{H} \lambda_{H} V-\mu_{0} V \\
\dot{I}_{H} & =\left[\pi_{H} V+(1-\delta) S\right] \lambda_{H}-\left(\mu_{0}+\gamma_{1}\right) I_{H} \\
\dot{C}_{H} & =\mu \omega v C_{H}+q \gamma_{1} I_{H}-\left(\mu_{0}+d_{H}+\gamma_{2}\right) C_{H} \\
\dot{R}_{H} & =\gamma_{1}(1-q) I_{H}+\gamma_{2} C_{H}-\mu_{0} R_{H}
\end{aligned}\right.
$$


where $\lambda_{H}=\beta_{H} \frac{I_{H}+\eta C_{H}}{N}$ with $N=S+V+I_{H}+C_{H}+R_{H}$. It should be pointed out that the HBV-only model is a slight modification of HBV models proposed in Refs. [11-14]. In the model (3.1), we have assumed that the vaccine-induced immunity acquired by vaccinated individuals is suppose to wane and the way for vaccinated individuals to acquire HBV infection is through the waning of vaccine-induced protection, and individuals therefore enter the HBV acute infection class. This is no the case of the models proposed in Refs. [11-14] where the authors have supposed that when the vaccine-induced immunity wanes, vaccinated individuals return to the susceptible before acquire HBV infection.

The feasible region for this sub-model is

$$
\Omega_{H}=\left\{\left(S, V, I_{H}, C_{H}, R_{H}\right) \in \mathbb{R}_{\geq 0}^{5}, \quad S+V+I_{H}+C_{H}+R_{H} \leq \frac{\Lambda+\mu}{\mu_{0}}\right\} .
$$

Next, we show that the region $\Omega_{H}$ is positively invariant, so that it is sufficient to consider the dynamics of the above model in $\Omega_{H}$.

Adding all equations in (3.1) gives

$$
\dot{N}=\Lambda+\mu-\mu_{0} N-d_{H} C_{H} .
$$

Since the right hand side of the above equation is bounded by $\Lambda+\mu-\mu_{0} N(t)$, it follows that $\dot{N}<0$ if $N(t)>\frac{(\Lambda+\mu)}{\mu_{0}}$. Using a standard comparison theorem [47-49], it can be shown that $N(t) \leq N(0) e^{-\mu_{0} t}+\frac{(\Lambda+\mu)}{\mu_{0}}\left(1-e^{-\mu_{0} t}\right)$. Thus, $\Omega_{H}$ is positively-invariant. If $N(0)>\frac{\Lambda+\mu}{\mu_{0}}$, then, either the solution enters $\Omega_{H}$ in finite time, or $N(t)$ approaches $\frac{\Lambda+\mu}{\mu_{0}}$ asymptotically, and the infected variables $I_{H}, C_{H}$ and $R_{H}$ approach zero. Hence, $\Omega_{H}$ is attracting.

One of the most important concerns in the analysis of epidemiological models is the determination of the asymptotic behavior of their solutions which is usually based on the stability of the associated equilibria [50]. These models typically consist of a disease-free equilibrium and at least one endemic equilibrium. The local stability of the disease-free equilibrium (DFE) is determined based on a threshold parameter, known as the basic reproductive number [51, 52].

\subsubsection{Local stability of the disease-free equilibrium (DFE) of the HBV-only model}

The DFE of the HBV-only model (3.1) is given by,

$$
Q_{0}^{H}=\left(S_{0}, V_{0}, 0,0,0\right),
$$

where

$$
S_{0}=\frac{\Lambda+\mu \omega}{\mu_{0}+\delta} \quad \text { and } \quad V_{0}=\frac{\delta(\mu+\Lambda)+\mu \mu_{0}(1-\omega)}{\mu_{0}\left(\mu_{0}+\delta\right)} .
$$

The local stability of the DFE of the HBV-onlymodel is determined by its basic reproduction number $\mathcal{R}_{0}^{H}$, which is computed using the next generation operator method proposed by van den Driessche and Watmough [52]. 
Using the notations in [52], the matrices $F$ and $V$, for the new infection and the remaining transfer, are, respectively, given by

$$
F=\frac{\beta_{H}}{N_{0}}\left[\pi_{H} V_{0}+(1-\delta) S_{0}\right]\left[\begin{array}{ccc}
1 & \eta & 0 \\
0 & 0 & 0 \\
0 & 0 & 0
\end{array}\right] \quad \text { and } \quad V=\left[\begin{array}{ccc}
\mu_{0}+\gamma_{1} & 0 & 0 \\
-q \gamma_{1} & A & 0 \\
-\gamma_{1}(1-q) & -\gamma_{2} & \mu_{0}
\end{array}\right]
$$

where $N_{0}=S_{0}+V_{0}$ and $A=\mu_{0}+d_{H}+\gamma_{2}-\mu \omega v$. Then, the basic reproduction ratio is defined as the dominant eigenvalue of the next generation matrix, $F V^{-1}$ :

$$
\mathcal{R}_{0}^{H}=\frac{\beta_{H}\left(A+\eta q \gamma_{1}\right)\left[\pi_{H}\left[\delta(\mu+\Lambda)+\mu \mu_{0}(1-\omega)\right]+\mu_{0}(1-\delta)(\Lambda+\mu \omega)\right]}{A\left(\mu_{0}+\delta\right)\left(\mu_{0}+\gamma_{1}\right)(\Lambda+\mu)} .
$$

We can also interpret $\mathcal{R}_{0}^{H}$ as the average number of secondary HBV acute and chronic infection cases produced by a HBV acute or chronic individual during his or her effective infectious period when introduced in a population of mostly HBV susceptible. The threshold quantity $\mathcal{R}_{0}^{H}$ is the reproduction number for HBV. Then, we claim the following result.

Lemma 1. : The DFE $Q_{0}^{H}$ of the HBV-only model is locally asymptotically stable (LAS) if $\mathcal{R}_{0}^{H}<1$, and unstable if $\mathcal{R}_{0}^{H}>1$.

Biologically speaking, Lemma 1 implies that HBV can be eliminated from the community (when $\mathcal{R}_{0}^{H}<1$ ) if the initial size of the sub-populations of the model is in the basin of attraction of $Q_{0}^{H}$.

Now let us analyze the basic reproduction ratio $\mathcal{R}_{0}^{H}$. The parameters $\omega$ and $\delta$ are important for the prevalence of HBV infection. They influence the dynamics of HBV, in particular the equilibrium states, including the states of susceptible, acute infective, and carriers. It is evident from (3.3) that

$$
\lim _{\delta \rightarrow 1} \mathcal{R}_{0}^{H}=\frac{\beta_{H} \pi_{H}\left(A+\eta q \gamma_{1}\right)\left[\mu+\Lambda+\mu_{0} \mu(1-\omega)\right]}{A\left(\mu_{0}+1\right)\left(\mu_{0}+\gamma_{1}\right)(\Lambda+\mu)}>0 .
$$

Thus, a sufficiently effective HBV vaccination program can lead to effective disease control if it results in making the right-hand side of (3.4) less than unity, that is,

$$
\pi_{H}<\frac{A\left(\mu_{0}+1\right)\left(\mu_{0}+\gamma_{1}\right)(\Lambda+\mu)}{\beta_{H}\left(A+\eta q \gamma_{1}\right)\left[\mu+\Lambda+\mu_{0} \mu(1-\omega)\right]} .
$$

Further, sensitivity analysis on the vaccination parameter of susceptible individuals is carried out by computing the partial derivative of $\mathcal{R}_{0}^{H}$ with respect to $\delta$ yielding

$$
\frac{\partial \mathcal{R}_{0}^{H}}{\partial \delta}=-\frac{\beta_{H} \mu_{0}(\Lambda+\mu \omega)\left(A+\eta q \gamma_{1}\right)\left(\mu_{0}+1-\pi_{H}\right)}{A\left(\mu_{0}+\gamma_{1}\right)(\Lambda+\mu)\left(\mu_{0}+\delta\right)^{2}}<0 .
$$

Thus, increasing the vaccination parameter $\delta$ will have a positive impact in reducing the propagation of HBV in the community.

To find better control strategies for HBV infection, we would like to see what parameters can reduce the basic reproduction ratio $\mathcal{R}_{0}^{H}$ given by (3.3). 
Figure 2 presents the 3-D plot showing the effects of $\beta_{H}, 1-\omega$ and $\delta$ on the basic reproduction ratio $\mathcal{R}_{0}^{H}$. All other parameters are as in Table 3. From Fig. 2(a), we can see that $\mathcal{R}_{0}^{H}$ decreases if $\beta_{H}$ decreases (see Fig. 2(a)) even in the case of large values of $1-\omega$. This means that if the transmission coefficient $\beta_{H}$ is sufficiently small HBV infection could be eliminated even if $1-\omega=0$. Also, it clearly appears from Fig. 2(b) that $\mathcal{R}_{0}^{H}$ decreases if $\delta$ increases or $\beta_{H}$ decreases. However, it is difficult to control $\beta_{H}$. Figure 2(c) shows the effects of $\delta$ and $1-\omega$ on the basic reproduction ratio $\mathcal{R}_{0}^{H}$ when $\beta_{H}=5$. As expected, $\mathcal{R}_{0}^{H}$ might be smaller than 1 as long as $\delta$ and $1-\omega$ are large enough. Therefore, immunization of both newborns and susceptible individuals is an efficient intervention. Thus, combining immunization with reduction of contacts can reduce $\mathcal{R}_{0}^{H}$ to be less than 1 . Then, the optimal control strategy will be a combination of immunization of newborns, immunization of susceptible individuals (at least young adults), and reduction of contacts.

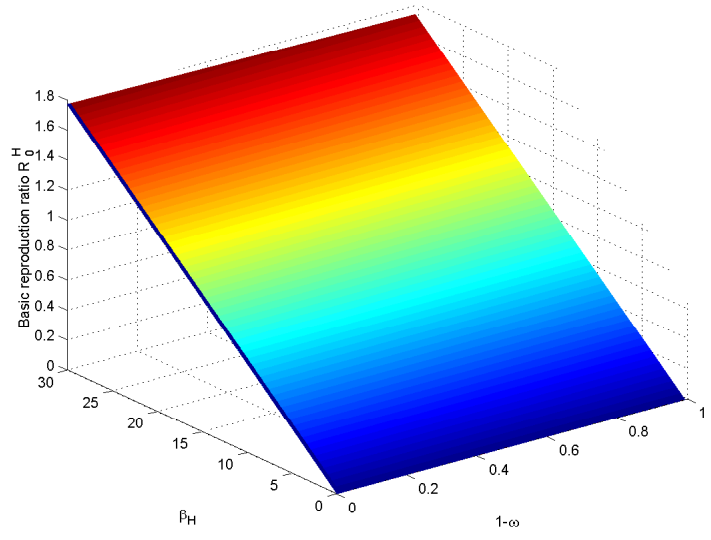

(a)

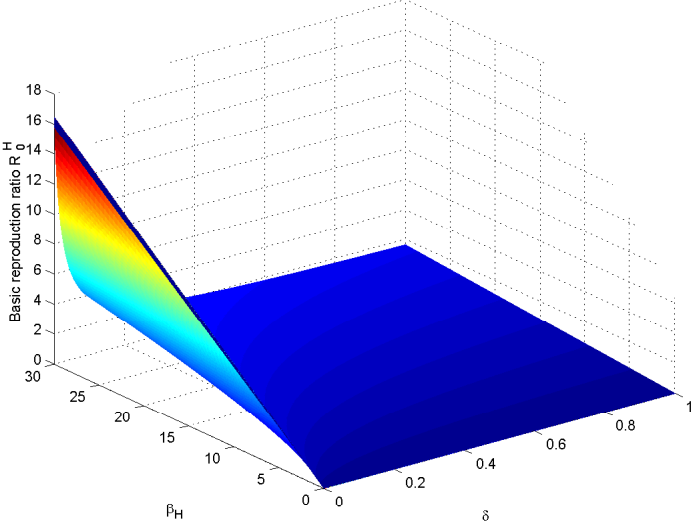

(b)

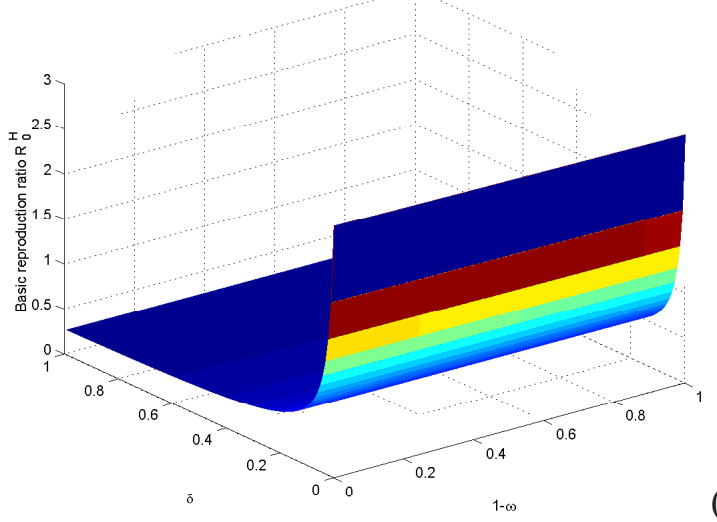

(c)

Figure 2: Graphs of the basic reproduction ratio $\mathcal{R}_{0}^{H}$ of system (3.1) in dependence on some parameters: (a) $\mathcal{R}_{0}^{H}$ in terms of $1-\omega$ and $\beta_{H}$, (b) $\mathcal{R}_{0}^{H}$ in terms of $\delta$ and $\beta_{H}$, and (c) $\mathcal{R}_{0}^{H}$ in terms of $1-\omega$ and $\delta$ when $\beta_{H}=5$. All other parameters are as in Table 3 . 


\subsubsection{Global stability of DFE of the HBV-only model}

We get the following result concerning the stability of the DFE $Q_{0}^{H}$ of system (3.1).

Theorem 2. The DFE of system (3.1), given by $Q_{0}^{H}$, is globally-asymptotically stable $(G A S)$ in $\Omega_{H}$ whenever $\mathcal{R}_{0}^{H} \leq 1$.

Proof: Consider the following LaSalle-Lyapunov candidate function:

$$
\mathcal{L}\left(I_{H}, C_{H}\right)=a_{1} I_{H}+a_{2} C_{H},
$$

where

$$
a_{1}=A \quad \text { and } \quad a_{2}=\frac{\beta_{H} \eta\left[\pi_{H} V_{0}+(1-\delta) S_{0}\right]}{S_{0}+V_{0}} .
$$

Its time derivative along the trajectories of system (3.1) satisfies

$$
\begin{aligned}
\dot{\mathcal{L}} & =a_{1} \dot{I}_{H}+a_{2} \dot{C}_{H} \\
& =a_{1}\left(\frac{\beta_{H}\left[\pi_{H} V+(1-\delta) S\right]}{N}\left(I_{H}+\eta C_{H}\right)-\left(\mu_{0}+\gamma_{1}\right) I_{H}\right)+a_{2}\left(q \gamma_{1} I_{H}-A C_{H}\right) .
\end{aligned}
$$

Since the state variables of the model when the HBV is endemic in the population do not exceed the state variables of the model in a population free of $\mathrm{HBV}$, one has that $\frac{\left[\pi_{H} V+(1-\delta) S\right]}{N} \leq$ $\frac{\left[\pi_{H} V_{0}+(1-\delta) S_{0}\right]}{S_{0}+V_{0}}$, one has

$$
\begin{aligned}
\dot{\mathcal{L}} & \leq\left(\frac{a_{1} \beta_{H}\left[\pi_{H} V_{0}+(1-\delta) S_{0}\right]}{S_{0}+V_{0}}-a_{1}\left(\mu_{0}+\gamma_{1}\right)+a_{2} q \gamma_{1}\right) I_{H} \\
& +\left(\frac{a_{1} \beta_{H} \eta\left[\pi_{H} V_{0}+(1-\delta) S_{0}\right]}{S_{0}+V_{0}}-a_{2} A\right) C_{H} .
\end{aligned}
$$

Now, plugging the positive constants $a_{1}$ and $a_{2}$ given in Eq. (3.6) into Eq. (3.8), one finally obtains

$$
\begin{aligned}
\dot{\mathcal{L}} & \leq\left(\frac{\beta_{H}\left(A+\eta q \gamma_{1}\right)\left[\pi_{H} V_{0}+(1-\delta) S_{0}\right]}{S_{0}+V_{0}}-A\left(\mu_{0}+\gamma_{1}\right)\right) I_{H}, \\
& \leq A\left(\mu_{0}+\gamma_{1}\right)\left(\frac{\beta_{H}\left(A+\eta q \gamma_{1}\right)\left[\pi_{H} V_{0}+(1-\delta) S_{0}\right]}{A\left(S_{0}+V_{0}\right)\left(\mu_{0}+\gamma_{1}\right)}-1\right) I_{H}, \\
& \leq A\left(\mu_{0}+\gamma_{1}\right)\left(\mathcal{R}_{0}^{H}-1\right) I_{H} .
\end{aligned}
$$

Thus, $\dot{\mathcal{L}} \leq 0$ when $\mathcal{R}_{0}^{H} \leq 1$. By LaSalle's invariance principle, the largest invariant set in $\Omega_{H}$ contained in $\left\{\left(S, V, I_{H}, C_{H}, R_{H}\right) \in \mathbb{R}_{\geq 0}^{5} \quad \dot{\mathcal{L}}=0\right\}$ is reduced to the DFE. This proves the global asymptotic stability on $\Omega_{H}$ ( [55, Theorem 3.7.11, page 346]). Since $\Omega_{H}$ is absorbing, this proves 
the global asymptotic stability on the nonnegative orthant when $\mathcal{R}_{0}^{H} \leq 1$. We stress the need to consider a positively invariant compact set to establish the stability of the DFE since the function $\mathcal{L}$ is not positive definite. Generally the LaSalle's invariance principle only proves the attractivity of the equilibrium. Considering $\Omega_{H}$ permits us to conclude for the stability [53-55]. This fact is often overlooked in the literature using the LaSalle's invariance principle. This concludes the proof.

Figure 3 shows simulation results converging to the disease free equilibrium for the total number of $\mathrm{HBV}$ infected individuals using various initial conditions when $\beta_{H}=10$ (so that $\mathcal{R}_{0}^{H}=0.5805$ ). All other parameters are as in Table 3. This result shows that HBV can be eliminated from the community if the associated threshold quantity, $\mathcal{R}_{0}^{H}$, can be brought to a value less than unity.

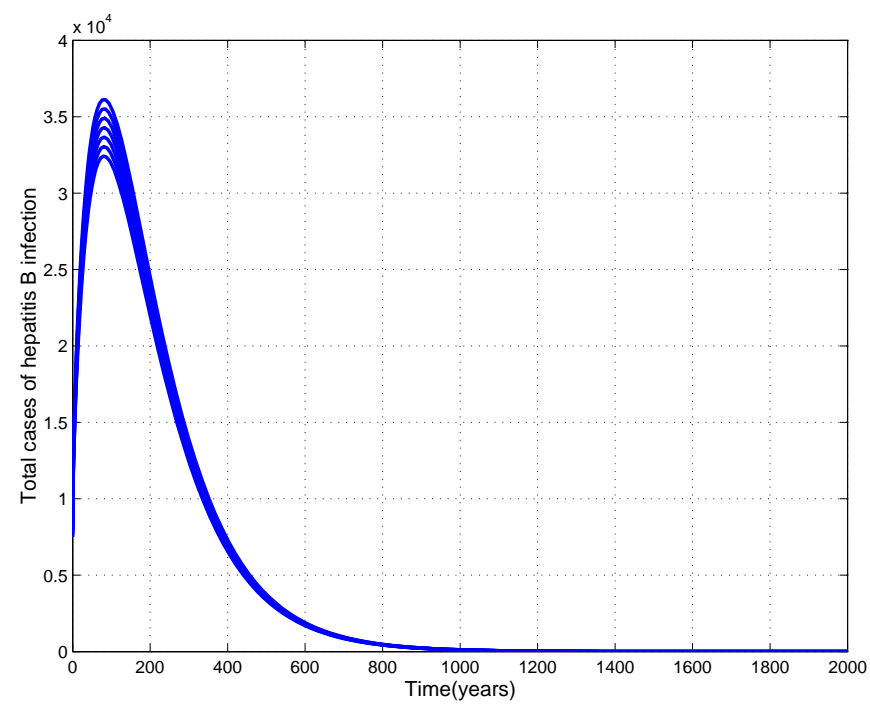

Figure 3: Time series of HBV-only model (3.1) showing the total number of the HBV infected individuals as a function of time, using various initial conditions for $\beta_{H}=10$ (so that $\mathcal{R}_{0}^{H}=$ 0.5805). All other parameters are as in Table 3.

\subsubsection{Existence and stability of the endemic equilibrium}

Because $R_{H}$ appears only in the fifth equation of the model, we can discuss the steady states without this variable. To find conditions for the existence of an endemic equilibrium for which $\mathrm{HBV}$ is endemic in the population (i.e., at least one of $I_{H}^{*}$ and $C_{H}^{*}$ is non-zero), denoted by $Q_{H}^{*}=$ $\left(S_{H}^{*}, V_{H}^{*}, I_{H}^{*}, C_{H}^{*}\right)$, equations in (3.1) are solved in terms of the force of infection at the steady-state $\left(\lambda_{H}^{*}\right)$ yields

$$
\lambda_{H}^{*}=\frac{\beta_{H}\left(I_{H}^{*}+\eta C_{H}^{*}\right)}{N_{H}^{*}} .
$$


Setting the right hand sides of the model to zero (and noting that $\lambda_{H}=\lambda_{H}^{*}$ at equilibrium) gives

$$
\left\{\begin{array}{l}
\Lambda+\mu \omega\left(1-v C_{H}^{*}\right)-(1-\delta) \lambda_{H}^{*} S_{H}^{*}-\left(\delta+\mu_{0}\right) S_{H}^{*}=0 \\
\mu(1-\omega)+\delta S_{H}^{*}-\pi_{H} \lambda_{H}^{*} V^{*}-\mu_{0} V^{*}=0 \\
\left(\pi_{H} V_{H}^{*}+(1-\delta) S_{H}^{*}\right) \lambda_{H}^{*}-\left(\mu_{0}+\gamma_{1}\right) I_{H}^{*}=0 \\
\mu \omega v C_{H}^{*}+q \gamma_{1} I_{H}^{*}-\left(\mu_{0}+d_{H}+\gamma_{2}\right) C_{H}^{*}=0 .
\end{array}\right.
$$

Solving the above equations in (3.11) at steady state gives

$$
\left\{\begin{aligned}
I_{H}^{*} & =\frac{A(\Lambda+\mu) \lambda_{H}^{*}}{\beta_{H} \mu_{0}\left(A+\eta q \gamma_{1}\right)+d_{H} q \gamma_{1} \lambda_{H}^{*}}, \\
C_{H}^{*} & =\frac{q \gamma_{1}(\Lambda+\mu) \lambda_{H}^{*}}{\beta_{H} \mu_{0}\left(A+\eta q \gamma_{1}\right)+d_{H} q \gamma_{1} \lambda_{H}^{*}}, \\
S_{H}^{*} & =\frac{1}{(1-\delta) \lambda_{H}^{*}+\delta+\mu_{0}}\left[\Lambda+\mu \omega\left(1-\frac{q \gamma_{1} v(\Lambda+\mu) \lambda_{H}^{*}}{\beta_{H} \mu_{0}\left(A+\eta q \gamma_{1}\right)+d_{H} q \gamma_{1} \lambda_{H}^{*}}\right)\right], \\
V_{H}^{*} & =\frac{1}{\pi_{H} \lambda_{H}^{*}+\mu_{0}}[\mu(1-\omega) \\
& \left.+\frac{\delta}{(1-\delta) \lambda_{H}^{*}+\delta+\mu_{0}}\left[\Lambda+\mu \omega\left(1-\frac{q \gamma_{1} v(\Lambda+\mu) \lambda_{H}^{*}}{\beta_{H} \mu_{0}\left(A+\eta q \gamma_{1}\right)+d_{H} q \gamma_{1} \lambda_{H}^{*}}\right)\right]\right] .
\end{aligned}\right.
$$

Substituting (3.12) in (3.10), it can be shown that the non-zero equilibria of system (3.1) satisfy the following quadratic equation in term of $\lambda_{H}^{*}$ :

$$
b_{2}\left(\lambda_{H}^{*}\right)^{2}+b_{1} \lambda_{H}^{*}+b_{0}=0,
$$

where

$$
\begin{aligned}
b_{2} & =(1-\delta)(\Lambda+\mu)\left[\pi_{H}\left[A\left(\mu_{0}+\gamma_{1}\right)-d_{H} q \gamma_{1}\right]+\mu \omega v q \gamma_{1}(1-\delta)\right], \\
b_{1} & =-\pi_{H} \mu(1-\omega)\left[\beta_{H} \mu_{0}(1-\delta)\left(A+\eta q \gamma_{1}\right)+d_{H} q \gamma_{1}\left(\mu_{0}+\delta\right)\right] \\
& -d_{H} q \gamma_{1} \pi_{H} \delta(\Lambda+\mu \omega)+\pi_{H} \delta \mu v q \gamma_{1}(\Lambda+\mu) \\
& -(1-\delta)(\Lambda+\mu \omega)\left[\pi_{H} \beta_{H} \mu_{0}\left(A+\eta q \gamma_{1}\right)+\mu_{0} d_{H} q \gamma_{1}\right] \\
& +\mu \omega v q \gamma_{1}(1-\delta)\left(\mu_{0}+\delta\right)(\Lambda+\mu) \\
& +A\left(\mu_{0}+\gamma_{1}\right)(\Lambda+\mu)\left[\pi_{H}\left(\mu_{0}+\delta\right)+\mu_{0}(1-\delta)\right] \\
b_{0} & =A \mu_{0}\left(\mu_{0}+\gamma_{1}\right)\left(\mu_{0}+\delta\right)(\Lambda+\mu)\left(1-\mathcal{R}_{0}^{H}\right) .
\end{aligned}
$$


It is worth noting that the coefficient $b_{2}$ is always positive and $b_{0}$ is positive if $\mathcal{R}_{0}^{H}$ is less than unity, and negative if $\mathcal{R}_{0}^{H}$ is greater than unity. Thus, the number of possible real roots of Eq. (3.13) can have depends on the signs of $b_{2}, b_{1}$ and $b_{0}$. This can be analyzed using the Descartes Rule of Signs on the polynomial

$$
f\left(\lambda_{H}^{*}\right)=b_{2}\left(\lambda_{H}^{*}\right)^{2}+b_{1} \lambda_{H}^{*}+b_{0} .
$$

Since $\lambda_{H}^{*} \in\left[0, \beta_{H}(1+\eta)\right]$, one can easily verify that

$$
\begin{array}{ll}
f(0) & =b_{0}=A \mu_{0}\left(\mu_{0}+\gamma_{1}\right)\left(\mu_{0}+\delta\right)(\Lambda+\mu)\left(1-\mathcal{R}_{0}^{H}\right), \\
f\left(\beta_{H}(1+\eta)\right) & =b_{2}\left(\beta_{H}(1+\eta)\right)^{2}+b_{1}\left(\beta_{H}(1+\eta)\right)+b_{0} .
\end{array}
$$

A simple calculation proves that $f\left(\beta_{H}(1+\eta)>0\right.$. It is now a trivial matter to see that $f(0)<0$ when $\mathcal{R}_{0}^{H}>1$. The existence follows from the intermediate value theorem. Now, $f\left(\lambda_{H}^{*}\right)$ is monotone increasing, so that $f\left(\lambda_{H}^{*}\right)=0$ has only one positive root in the interval $\left[0, \beta_{H}(1+\eta)\right]$. Thus, we have established the following result.

Lemma 3. When $\mathcal{R}_{0}^{H}>1$, the model (3.1) has a unique endemic equilibrium $Q_{H}^{*}=\left(S_{H}^{*}, V_{H}^{*}\right.$, $\left.I_{H}^{*}, C_{H}^{*}, R_{H}^{*}\right)$ with $S_{H}^{*}, V_{H}^{*}, I_{H}^{*}, C_{H}^{*}$ and $R_{H}^{*}$ all nonnegative.

Now, let us study the stability of the endemic equilibrium guaranteed by Lemma 3. Using standard linearization of the HBV-only model around the endemic equilibrium is laborious and not really tractable mathematically. Here, the center manifold theory [56], as described in [57] (Theorem 4.1), will be used to establish the local asymptotic stability of the endemic equilibrium (see also [52, 58]).

Then, we claim the following result (the proof is given in Appendix A):

Lemma 4. The unique endemic equilibrium guaranteed by Lemma 3 is LAS for $\mathcal{R}_{0}^{H}$ near 1.

It should be pointed out that this result holds for $\mathcal{R}_{0}^{H}>1$ but close to 1 .

Figure 4 shows convergence to the endemic equilibrium for the total number of infected individuals using various initial conditions when $\beta_{H}=20$ (so that $\mathcal{R}_{0}^{H}=1.1609$ ). All other parameters are as in Table 3.

In summary, the HBV-only model (3.1) has a globally-asymptotically stable DFE whenever $\mathcal{R}_{0}^{H} \leq 1$, and a unique endemic equilibrium point whenever $\mathcal{R}_{0}^{H}>1$. The unique endemic equilibrium point is LAS at least near $\mathcal{R}_{0}^{H}=1$. The dynamics of TB-only is also explored as below.

\subsection{TB-only sub-model}

The high prevalence of tuberculosis in developing countries and recent resurgence of tuberculosis in many developed countries suggest that current control strategies are suboptimal. More than 36 million patients have been successfully treated via the World Health Organization strategy for tuberculosis control since 1995. Despite predictions of a decline in global incidence, the number of new cases continues to grow, approaching 10 million in 2010 [1,2]. This rise has been attributed 


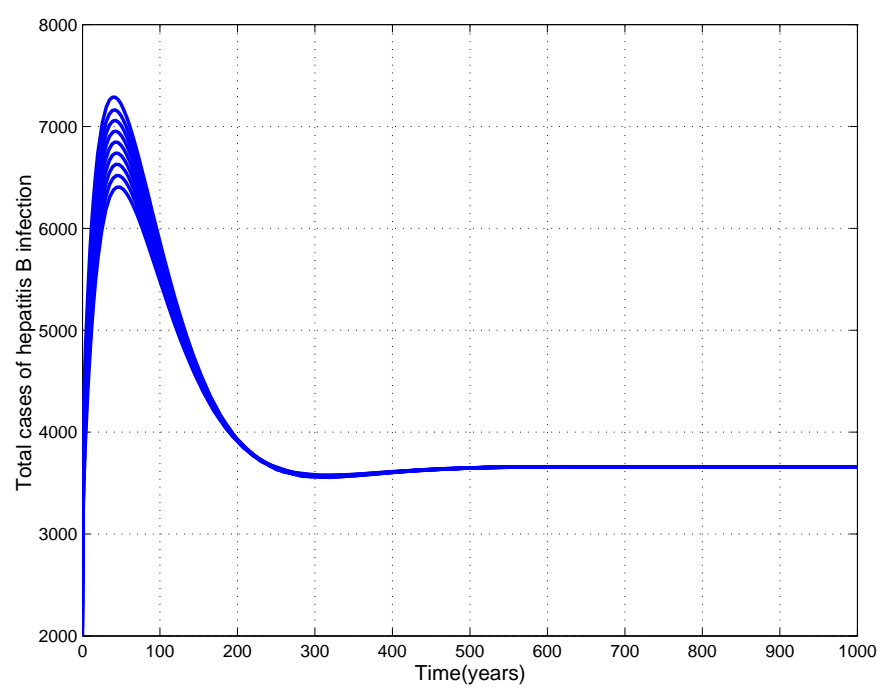

Figure 4: Time series of HBV-only model (3.1) showing the total number of infected individuals as a function of time, using various initial conditions for $\beta_{H}=20$ (so that $\mathcal{R}_{0}^{H}=1.1609$ ). All other parameters are as in Table 3.

to the spread of HIV, the collapse of public health programs and the emergence of drug-resistant strains of $M$. tuberculosis. Another issue that is essential to the epidemiology of TB is the exogenous re-infection, where a latently-infected individual acquires a new infection from another infectious (see [34, 61] and references therein).

The TB-only model is obtained by setting $V=I_{H}=C_{H}=R_{H}=E_{T I}=I_{T I}=E_{T C}=$ $I_{T C}=E_{T R}=I_{T R}=0$ and $\delta=\omega=0$, which gives,

$$
\left\{\begin{array}{l}
\dot{S}=\Lambda-\lambda_{T} S-\mu_{0} S \\
\dot{E}_{T}=(1-p) \lambda_{T} S+r_{2} I_{T}-\left(1-r_{1}\right) \sigma \lambda_{T} E_{T}-\left[\mu_{0}+k\left(1-r_{1}\right)\right] E_{T}, \\
\dot{I}_{T}=p \lambda_{T} S+\left(1-r_{1}\right)\left(k+\sigma \lambda_{T}\right) E_{T}-\left(\mu_{0}+d_{T}+r_{2}\right) I_{T},
\end{array}\right.
$$

where $\lambda_{T}=\beta_{T} \frac{I_{T}}{N}$ and $N=S+E_{T}+I_{T}$. Note that since the population is free of HBV, there is no HBV infected individuals who can transmit HBV infection to susceptible individuals. Therefore, it is not necessary to vaccine people with respect to HBV. So, in this case, one has $\delta=\omega=0$. We point out that the TB-only model (3.15) is an extension of the model proposed by Kirchner et al. [66], which, in our opinion, captures the essentials of Mycobacterium tuberculosis transmission. In our model, exogenous reinfections have been take into account.

The feasible region for this sub-model is

$$
\Omega_{T}=\left\{\left(S, E_{T}, I_{T}\right) \in \mathbb{R}_{\geq 0}^{3}, \quad N(t) \leq \frac{\Lambda}{\mu_{0}}\right\}
$$


Likewise $\Omega_{H}$ above, it can be shown that all solutions of system (3.15) starting in $\Omega_{T}$ remain in $\Omega_{T}$ for all $t \geq 0$. Thus, $\Omega_{T}$ is positively invariant (hence, it is sufficient to consider the dynamics of (3.15) in $\Omega_{T}$ ).

\subsubsection{Local stability of the DFE of the TB-only model}

The TB-only model (3.15) has a DFE given by

$$
Q_{0}^{T}=\left(\frac{\Lambda}{\mu_{0}}, 0,0\right)
$$

The stability of this equilibrium will be investigated using the next generation operator method $[51,52]$. Using the notations in [52] on system (3.15), the matrices $F$ and $V$, for the new infection terms and the remaining transfer terms are, respectively, given by

$$
F=\left[\begin{array}{cc}
0 & \beta_{T}(1-p) \\
0 & \beta_{T} p
\end{array}\right] \quad \text { and } \quad V=\left[\begin{array}{cc}
A_{1} & -r_{2} \\
-k\left(1-r_{1}\right) & A_{2}
\end{array}\right]
$$

where $A_{1}=\mu_{0}+k\left(1-r_{1}\right)$ and $A_{2}=\mu_{0}+d_{T}+r_{2}$. The spectral radius or the largest eigenvalue of its next generation operator is given by

$$
\mathcal{R}_{0}^{T}=\frac{\beta_{T}\left[p \mu_{0}+k\left(1-r_{1}\right)\right]}{\mu_{0}\left(\mu_{0}+d_{T}+r_{2}\right)+k\left(1-r_{1}\right)\left(\mu_{0}+d_{T}\right)} .
$$

Thus, from Theorem 2 in [52], the following result holds.

Lemma 5. The DFE of the TB-only model given by the subsystem (3.15) is LAS if $\mathcal{R}_{0}^{T}<1$, and unstable if $\mathcal{R}_{0}^{T}>1$.

Now let us analyze the basic reproduction ratio $\mathcal{R}_{0}^{T}$. The objective here is to determine, using the threshold quantity $\mathcal{R}_{0}^{T}$, whether or not chemoprophylaxis of latently infected individuals and treatment of infectious lead to an effective control or elimination of TB in the population. Figure 5 shows the effects of the chemoprophylaxis rate $r_{1}$ and the treatment rate $r_{2}$ on the basic reproduction ratio $\mathcal{R}_{0}^{T}$ when $\beta_{T}=0.5$. All other parameters are as in Table 3. As expected, when $r_{1}$ is fixed, the basic reproduction ratio, $\mathcal{R}_{0}^{T}$, decreases as $r_{2}$ increases and vice versa. Then, combining the chemoprophylaxis of latently infected individuals and treatment of infectious can reduced $\mathcal{R}_{0}^{T}$ to less than unity. Therefore, the best control strategy will be the chemoprophylaxis of latently infected individuals and treatment of infectious or a combinaison of chemoprophylaxis and treatment.

The threshold quantity $\mathcal{R}_{0}^{T}$ is the reproduction number for TB. Biologically speaking, Lemma 4 implies that TB can be eliminated from the community (when $\mathcal{R}_{0}^{T}<1$ ) if the initial size of the sub-populations of the model is in the basin of attraction of $Q_{0}^{T}$. Since TB models may undergo the phenomenon of backward bifurcation (see [58] and references therein), it is instructive to determine whether the present sub-model exhibits this phenomenon. 


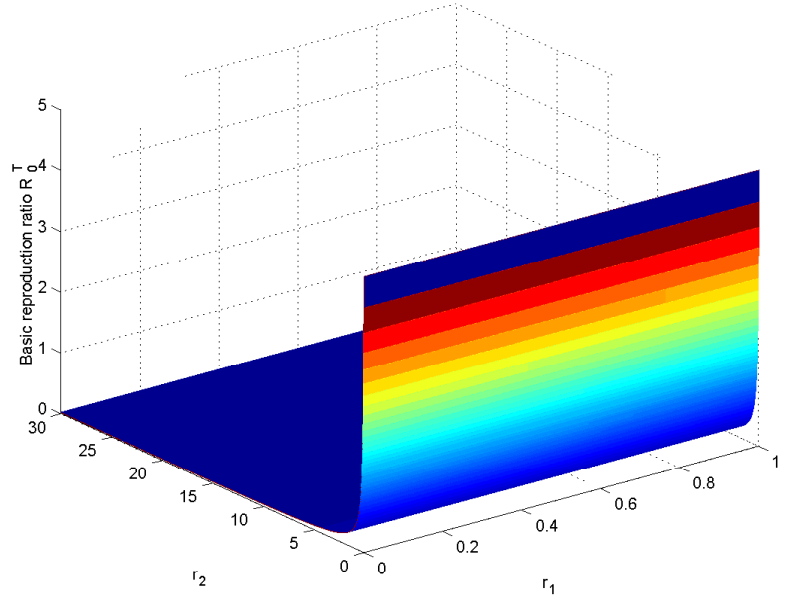

Figure 5: Graphs of the basic reproduction ratio $\mathcal{R}_{0}^{T}$ of system (3.15) in terms of $r_{1}$ and $r_{2}$ when $\beta_{T}=0.5$. All other parameters are as in Table 3 .

\subsubsection{Existence of backward bifurcation}

System (3.15) has one disease-free equilibrium, $Q_{0}^{T}=\left(S_{0}, 0,0\right)$ and one or two endemic equilibria of the form $Q_{T}^{*}=\left(S_{T}^{*}, E_{T}^{*}, I_{T}^{*}\right)$. On the other hand, from Lemma 4, the DFE of the TBonly model is locally asymptotically stable (LAS) if $\mathcal{R}_{0}^{T}<1$. However, this equilibrium may not be globally asymptotically stable (GAS) in $\Omega_{T}$ for $\mathcal{R}_{0}^{T}<1$, owing to the possibility of backward bifurcation, where the stable DFE co-exists with a stable endemic equilibrium when $\mathcal{R}_{0}^{T}<1$ (see for instance [58-65]) and references therein). In general, substitution of standard incidence with mass action incidence in some differential models removes their backward bifurcation property [63]. The standard incidence together with a large disease induced death rate are the driving force herein. The public health implication of backward bifurcation is that the classical requirement of having the basic reproductive number less than unity, although necessary, is no longer sufficient for curtailing the outbreak of the disease [64]. The possibility of this phenomenon in (3.15) is investigated below. Solving the TB-only model at an arbitrary equilibrium $Q_{T}^{*}=\left(S_{T}^{*}, E_{T}^{*}, I_{T}^{*}\right)$ gives

$$
\left\{\begin{array}{l}
\Lambda-\lambda_{T}^{*} S^{*}-\mu_{0} S_{T}^{*}=0, \\
(1-p) \lambda_{T}^{*} S^{*}-\sigma\left(1-r_{1}\right) \lambda_{T}^{*} E_{T}^{*}+r_{2} I_{T}^{*}-A_{1} E_{T}^{*}=0, \\
p \lambda_{T}^{*} S^{*}+\sigma\left(1-r_{1}\right) \lambda_{T}^{*} E_{T}^{*}+k\left(1-r_{1}\right) E_{T}^{*}-A_{2} I_{T}^{*}=0,
\end{array}\right.
$$

where $A_{1}$ and $A_{2}$ are defined as in Eq. (3.17), and

$$
\lambda_{T}^{*}=\beta_{T} \frac{I_{T}^{*}}{N^{*}},
$$


is the force of infection at the steady state. Solving the equations in (3.18) at the steady state gives

$$
\begin{aligned}
S_{T}^{*} & =\frac{\Lambda}{\mu_{0}+\lambda_{T}^{*}}, \\
E_{T}^{*} & =\frac{\lambda_{T}^{*} S^{*}\left[(1-p) A_{2}+r_{2} p\right]}{A_{1} A_{2}-r_{2} k\left(1-r_{1}\right)+\sigma\left(1-r_{1}\right)\left(A_{2}-r_{2}\right) \lambda_{T}^{*}}, \\
I_{T}^{*} & =\frac{\lambda_{T}^{*} S^{*}\left[(1-p) k\left(1-r_{1}\right)+p A_{1}+\sigma\left(1-r_{1}\right) \lambda_{T}^{*}\right]}{A_{1} A_{2}-r_{2} k\left(1-r_{1}\right)+\sigma\left(1-r_{1}\right)\left(A_{2}-r_{2}\right) \lambda_{T}^{*}} .
\end{aligned}
$$

Substituting the expressions in (3.20) in Eq. (3.19), it can be shown that the non-zero equilibria of the system (3.15) satisfy the following quadratic equation in term of $\lambda_{T}^{*}$ :

$$
c_{2}\left(\lambda_{T}^{*}\right)^{2}+c_{1}\left(\lambda_{T}^{*}\right)+c_{0}=0
$$

where

$$
\begin{aligned}
& c_{2}=\sigma\left(1-r_{1}\right), \\
& c_{1}=\sigma\left(1-r_{1}\right)\left(\mu_{0}+d_{T}-\beta_{T}\right)+(1-p)\left(\mu_{0}+d_{T}\right)+r_{2}+p \mu_{0}+k\left(1-r_{1}\right), \\
& c_{0}=\left[\left[\mu_{0}+k\left(1-r_{1}\right)\right]\left(\mu_{0}+d_{T}\right)+r_{2} \mu_{0}\right]\left(1-\mathcal{R}_{0}^{T}\right) .
\end{aligned}
$$

Thus, positive endemic equilibria $Q_{T}^{*}$ are obtained by solving for $\lambda_{T}^{*}$ from the quadratic equation (3.21) and substituting the result (positive values of $\lambda_{T}^{*}$ ) into (3.19). Clearly, the coefficient $c_{2}$ of Eq. (3.21) is always positive, and $c_{0}$ is positive or negative if $\mathcal{R}_{0}^{T}$ is less than or greater than unity, respectively. Thus, the number of possible real roots of the polynomial (3.21) can have depends on the signs of $c_{2}, c_{1}$ and $c_{0}$. Then, the following result holds:

Lemma 6. System (3.15) with TB alone has

(i) a unique endemic equilibrium when $c_{0}<0$, i.e., $\mathcal{R}_{0}^{T}>1$;

(ii) a unique endemic equilibrium when $c_{1}<0$, and $c_{0}=0$ or $c_{1}^{2}-4 c_{2} c_{0}=0$,

(iii) two endemic equilibria when $c_{0}>0, c_{1}<0$ and $c_{1}^{2}-4 c_{2} c_{0}>0$;

(iv) no endemic equilibria in all other cases.

It should be pointed out that the case (iii) indicates the possibility of a backward bifurcation phenomenon (where locally asymptotically stable DFE co-exists with a locally asymptotically stable endemic equilibrium when $\mathcal{R}_{0}^{T}<1$, (see for instance, [58-64]) in system (3.15) with TB alone. To check this, the discriminant $c_{1}^{2}-4 c_{2} c_{0}$ is set to zero and solved for the critical value of $\mathcal{R}_{0}^{T}$, denoted by $\mathcal{R}_{c}^{T}$, given by

$$
\mathcal{R}_{c}^{T}=1-\frac{c_{1}^{2}}{4 c_{2} \mathcal{R}^{*}}
$$

where

$$
\mathcal{R}^{*}=\mu_{0}\left(\mu_{0}+d_{T}+r_{2}\right)+k\left(1-r_{1}\right)\left(\mu_{0}+d_{T}\right) .
$$




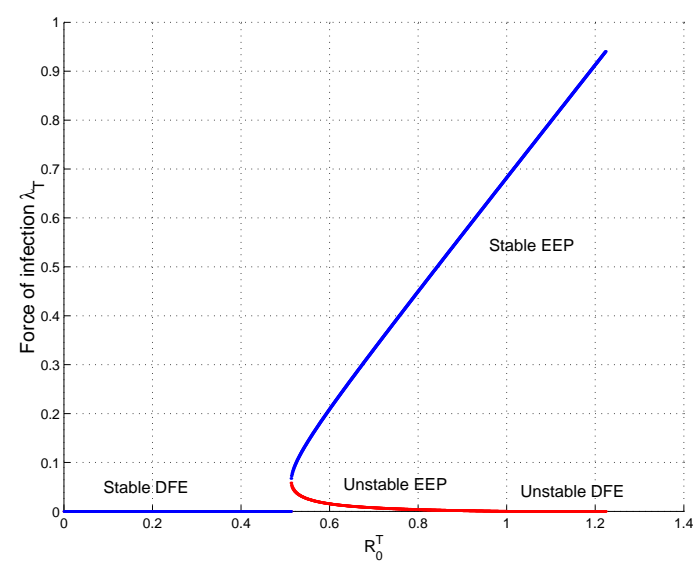

Figure 6: Bifurcation diagram for the model (3.15) where the notation EEP stands for endemic equilibrium point. All other parameters are as in Table 1.

The associated backward bifurcation diagram is depicted in Fig. 6.

The backward bifurcation phenomenon is also illustrated by simulating the system (3.15) with different initial conditions, parameters of Table 3 and $\beta_{T}=1$ (so that $\mathcal{R}_{0}^{T}=0.8733$ ). Figure 7 shows the convergence to both the disease-free equilibrium and the endemic equilibrium for the trajectories of system (3.15) when $\mathcal{R}_{0}^{T}<1$. In other words, from this figure, the profiles can converge to either the disease free equilibrium or an endemic equilibrium point, depending on the initial sizes of the population of the model (owing to the phenomenon of backward bifurcation).

Finally, it is worth stating that, unlike in the HBV-only model, the DFE of the TB-only model $\left(Q_{0}^{T}\right)$ is not globally-asymptotically stable when the associated reproductive number $\left(\mathcal{R}_{0}^{T}\right)$ is less than unity, owing to the phenomenon of backward bifurcation. Consequently, this study shows that the control of TB spread in a population when $\mathcal{R}_{0}^{T}<1$ depends on the initial sizes of the sub-populations of the TB-only model (3.15).

\section{Analysis of the full HBV-TB model}

Having analyzed the dynamics of the two sub-models, that is HBV-only and TB-only models, the full HBV-TB model (2.4) is now considered (the analysis is done in the positively invariant region $\Omega$ ).

\subsection{Local stability of the disease-free equilibrium}

The HBV-TB model (2.4) has a DFE given by

$$
Q_{0}=\left(S_{0}, V_{0}, 0,0,0,0,0,0,0,0,0,0,0\right) .
$$

where

$$
S_{0}=\frac{\Lambda+\mu \omega}{\mu_{0}+\delta} \quad \text { and } \quad V_{0}=\frac{\delta(\mu+\Lambda)+\mu \mu_{0}(1-\omega)}{\mu_{0}\left(\mu_{0}+\delta\right)}
$$



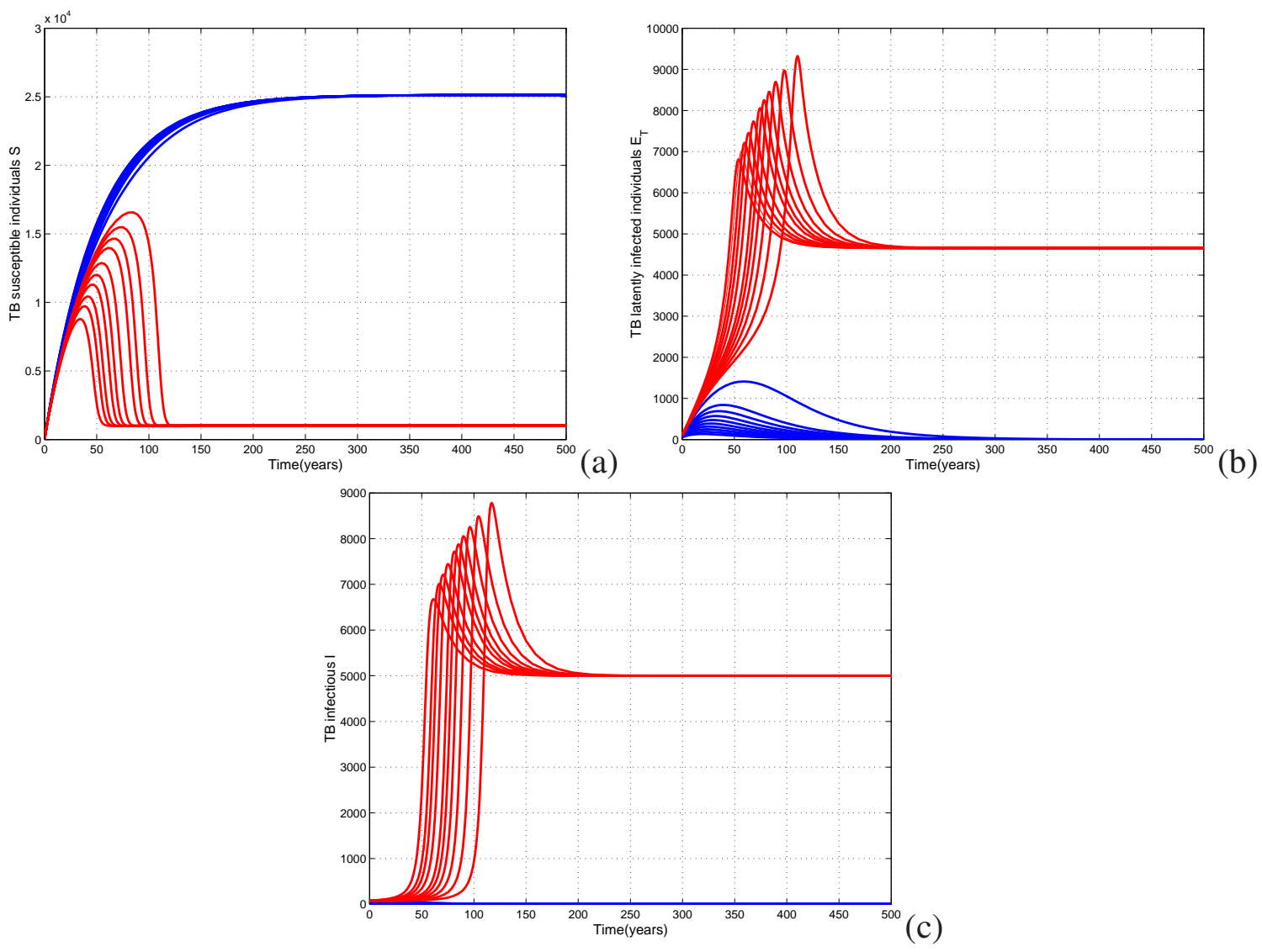

Figure 7: Simulation of system (3.15). Time series of (a) susceptible individuals, (b) latently infected individuals and (c) infectious when $\beta_{T}=1$ (so that $\mathcal{R}_{0}^{T}=0.8733$ ). All other parameters are as in Table 3.

The spectral radii of the next generation matrix $F V^{-1}$ are independent and given in terms of HBV and TB-only associated reproduction numbers (see details in Appendix B). It is therefore evident that the associated reproduction number for the full HBV-TB model denoted by $\mathcal{R}_{0}$ is given by

$$
\mathcal{R}_{0}=\max \left\{\mathcal{R}_{H}^{T}, \mathcal{R}_{0}^{H}\right\}
$$

where

$$
\begin{aligned}
\mathcal{R}_{0}^{H} & =\frac{\beta_{H}\left(A+\eta q \gamma_{1}\right)\left[\pi_{H}\left[\delta(\mu+\Lambda)+\mu \mu_{0}(1-\omega)\right]+\mu_{0}(1-\delta)(\Lambda+\mu \omega)\right]}{A\left(\mu_{0}+\delta\right)\left(\mu_{0}+\gamma_{1}\right)(\Lambda+\mu)}, \\
\mathcal{R}_{H}^{T} & =\frac{\beta_{T}\left[f \mu_{0}+k\left(1-r_{1}\right)\right] V_{0}}{\left(S_{0}+V_{0}\right)\left[\mu_{0}\left(\mu_{0}+d_{T}+r_{2}\right)+k\left(1-r_{1}\right)\left(\mu_{0}+d_{T}\right)\right]}+\frac{(1-\delta) S_{0}}{S_{0}+V_{0}} \mathcal{R}_{0}^{T},
\end{aligned}
$$

with $\mathcal{R}_{0}^{T}$ the basic reproduction ratio of the TB-only model defined in Eq. (3.17).

$\mathcal{R}_{H}^{T}$ gives the number of secondary TB infectious cases produced by a TB infectious individual during his or her effective infectious period when introduced in a population of mostly TB sus- 
ceptible. $\mathcal{R}_{0}^{H}$ is the HBV reproduction number in the absence of TB, which gives the number of secondary HBV acute or chronic individuals produced by an HBV acute or chronic individual (but not infected with TB) during his or her infectious period when introduced in a population of HBV susceptible who have no TB. Note that the reproductive numbers defined above do not involve the parameters associated with individuals who are co-infected with both TB and HBV. But, from Eq. (4.2), one can see that the basic reproduction ratio $\mathcal{R}_{H}^{T}$ depends on the parameters $\delta, \omega$ and $\mu$ which are parameters associated with the HBV disease. Additionally, using the expression of $\mathcal{R}_{H}^{T}$, it is evident that $\mathcal{R}_{H}^{T} \leq \mathcal{R}_{0}^{T}$.

A threshold condition for endemicity is given by $\mathcal{R}_{0}=1$ : the disease dies out if $\mathcal{R}_{0}<1$, and becomes endemic if $\mathcal{R}_{0}>1$. Then, we can claim the following result.

Lemma 7. The DFE $Q_{0}$ of system (2.4) is LAS if $\mathcal{R}_{0}<1$ and unstable if $\mathcal{R}_{0}>1$.

Figure 8 shows the 3-D plots showing the effects of $\beta_{T}, 1-\omega$ and $\delta$ on the basic reproduction ratio $\mathcal{R}_{H}^{T}$. All other parameters are as in Table 3 . It clearly appears that $\mathcal{R}_{H}^{T}$ decreases when $\beta_{T}$ decreases even if $1-\omega$ or $\delta$ has a large value. But, if the transmission coefficient $\beta_{T}$ is sufficiently small, TB could be eliminated even if $1-\omega=0$ or $\delta=0$. However, it is difficult to control $\beta_{T}$.
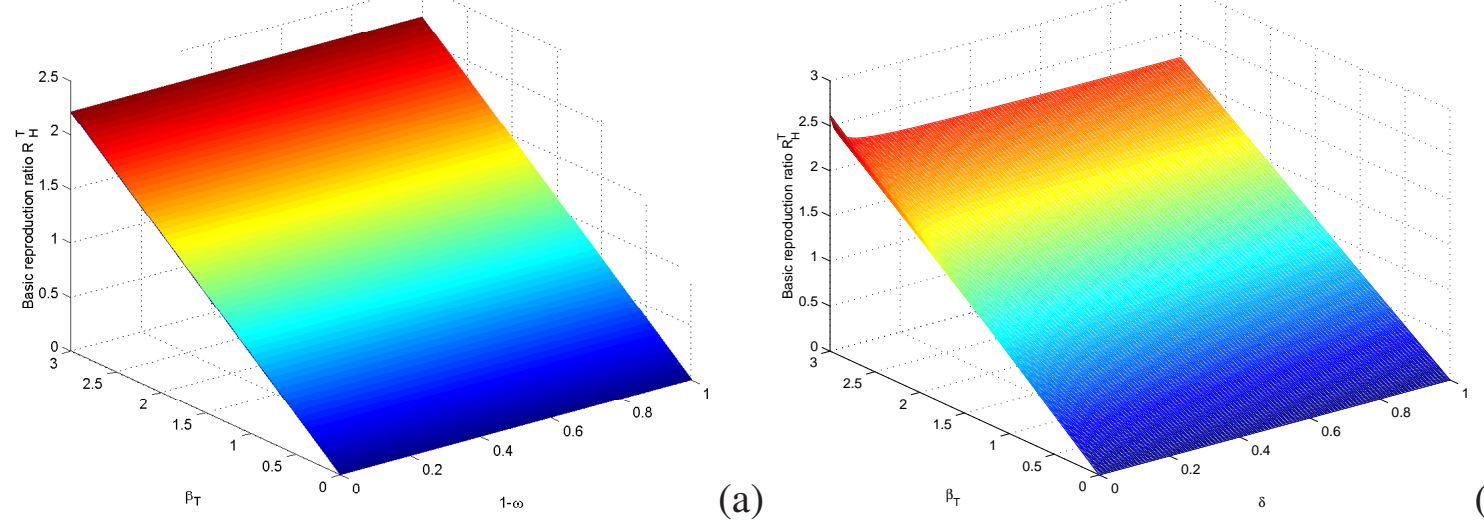

Figure 8: Graphs of the basic reproduction ratio $\mathcal{R}_{H}^{T}$ in terms of some parameters. (a) $\mathcal{R}_{H}^{T}$ in terms of $\beta_{T}$ and $1-\omega$, and (b) $\mathcal{R}_{H}^{T}$ of system (2.4) in terms of $\beta_{T}$ and $\delta$. All other parameters are as in Table 3.

It is worth noting that the DFE of the HBV-TB is not GAS, owing to the presence of the TBinduced backward bifurcation. Like in the case of the TB-only model (3.15), the full HBV-TB model (2.4) also undergoes a backward bifurcation phenomenon.

\subsubsection{Existence of boundaries equilibria and bifurcation parameter}

System (2.4) has three possible nonnegative boundary equilibria on $\Omega$ : one disease-free equilibrium, $Q_{0}=\left(S_{0}, V_{0}, 0,0,0,0,0,0,0,0,0,0,0\right)$ and one endemic equilibrium of the form $Q_{H}^{*}=$ $\left(S_{H}^{*}, V_{H}^{*}, I_{H}^{*}, C_{H}^{*}, R_{H}^{*}, 0,0,0,0,0,0,0,0,0\right)$, one or two endemic equilibria of the form $Q_{T}^{*}=$ $\left(S_{T}^{*}, 0,0,0,0, E_{T}^{*}, I_{T}^{*}, 0,0,0,0,0,0\right)$, and 
$Q^{*}=\left(S^{*}, V^{*}, I^{*}, C^{*}, R^{*}, E^{*}, I_{T}^{*}, E_{T I}^{*}, I_{T I}^{*}, E_{T C}^{*}, I_{T C}^{*}, E_{T R}^{*}, I_{T R}^{*}\right)$, corresponding, respectively, to states where only HBV, only TB, or both $\mathrm{HBV} / \mathrm{TB}$ are present. HBV-only (TB-free) equilibrium $Q_{H}^{*}$ exists if and only if $\mathcal{R}_{0}^{H}>1$ and the number of TB-only (HBV-free) equilibria $Q_{0}^{T}$ depends on the threshold parameters $\mathcal{R}_{0}^{T}$ and $\mathcal{R}_{H}^{T}$. Thus, there exists one TB-only (HBV-free) equilibrium if $\mathcal{R}_{0}^{T}>\mathcal{R}_{H}^{T}>1$ or $\mathcal{R}_{0}^{T}=1$ (cases (i) and (ii) of Lemma 6), one or two endemic equilibria if $\mathcal{R}_{H}^{T}<\mathcal{R}_{0}^{T}<1, c_{1}>0$ and $c_{1}^{2}-4 c_{0} c_{2}>0$ (case (iii) of Lemma 6)) is satisfied.

The TB-only model is shown to exhibit the phenomenon of backward bifurcation and consequently, the full HBV-TB model will exhibits the same feature. Therefore, we shall only derive the bifurcation parameters for the full model. By computing the eigenvalues of the Jacobian of the full HBV-TB model (2.4), it can be shown that $\mathcal{R}_{0}=\max \left\{\mathcal{R}_{H}^{T}, \mathcal{R}_{0}^{H}\right\}$, where $\mathcal{R}_{H}^{T}$ and $\mathcal{R}_{0}^{H}$ are defined as earlier. $\mathcal{R}_{0}=\max \left\{\mathcal{R}_{H}^{T}, \mathcal{R}_{0}^{H}\right\}$, therefore, there is no competitive exclusion and the two diseases enhance each other. Thus, when both reproduction numbers exceed unity (that is, $\mathcal{R}_{H}^{T}>1$ and $\mathcal{R}_{0}^{H}>1$ ), there is always a co-existence no matter which of the reproduction numbers is greater. Even though $\mathcal{R}_{0}$ does not combine the two reproduction numbers, it is worth not studying the two diseases in isolation because we will miss the dual HBV-TB only co-infection.

If $\max \left\{\mathcal{R}_{H}^{T}, \mathcal{R}_{0}^{H}\right\}=\mathcal{R}_{H}^{T}$, then, from Lemma 6 , the HBV-TB model exhibits backward bifurcation for values of $\mathcal{R}_{H}^{T}$ less than unity.

The backward bifurcation phenomenon of the full HBV-TB model (2.4) is illustrated by depicting time series plots, based on simulating the model (2.4) with various initial conditions when $\beta_{T}=1$ and $\beta_{H}=10$ (so that $\mathcal{R}_{0}^{H}=0.5805, \mathcal{R}_{0}^{T}=0.8733, \mathcal{R}_{H}^{T}=0.7363$ and $\mathcal{R}_{0}=0.7363$ ). They show convergence to either the DFE $\left(Q_{0}\right)$ or an endemic equilibrium (Figure 9).

Numerical simulations are also carried out to monitor the dynamics of the full model (2.4) using different initial conditions for the case where the associated basic reproduction number $\mathcal{R}_{H}^{T}$ does not exceed the unity. The results are reported in Figs. 10-11.

Figure 10 illustrates the solution profiles of the total of new TB cases, new HBV cases and dually-infected cases of the full model (2.4), using various initial conditions. Simulating the model using the parameter values in Table 3 with $\beta_{T}=0.1$ and $\beta_{H}=10$ (so that $\mathcal{R}_{0}^{H}=0.5805$, $\mathcal{R}_{0}^{T}=0.0873$ and $\mathcal{R}_{H}^{T}=0.0736$ ) shows convergence to the disease-free equilibrium (Fig. 10), in line with Lemma 7. Then, one can conclude that for smaller values of the $\mathcal{R}_{0}^{H}$ and $\mathcal{R}_{H}^{T}$, the DFE $\left(Q_{0}\right)$ is LAS. This has been verified via numerical simulations.

Now, we first select $\beta_{T}=1$ and $\beta_{H}=20$ such that $\left(\mathcal{R}_{0}^{H}=1.1609, \mathcal{R}_{0}^{T}=0.8733\right.$ and $\left.\mathcal{R}_{H}^{T}=0.7363\right)$. This corresponds to $\mathcal{R}_{0}^{H}>1$ and $\mathcal{R}_{H}^{T}<1$. From Fig. 11 , one can see that depending on the initial conditions, the trajectories of system (2.4) converge either to the diseasefree equilibrium or to the endemic equilibrium. This means that if $\mathcal{R}_{0}^{H}>1$, then it is possible that HBV disappears in the community (i.e., $Q_{0}^{H}$ is LAS) and it is also possible that TB becomes prevalent even if $\mathcal{R}_{H}^{T}<1$.

Numerical simulations for the case where the associated basic reproduction number $\mathcal{R}_{H}^{T}$ exceeds unity is carried out and depicted in Figs. 12-13. Choosing $\beta_{T}=2$ and $\beta_{H}=20$ (so that $\mathcal{R}_{0}^{H}=1.1609, \mathcal{R}_{0}^{T}=1.7467$ and $\mathcal{R}_{H}^{T}=1.4725$. This corresponds to $\mathcal{R}_{0}^{H}>1$ and $\mathcal{R}_{H}^{T}>1$. From Fig. 12, one can observe the convergence of the trajectories of the full model (2.4) to an endemic equilibrium. As expected, for $\mathcal{R}_{0}^{T}$ and $\mathcal{R}_{H}^{T}$ greater than unity, there is always co-existence of the two diseases no matter which of the reproduction numbers is greater. 

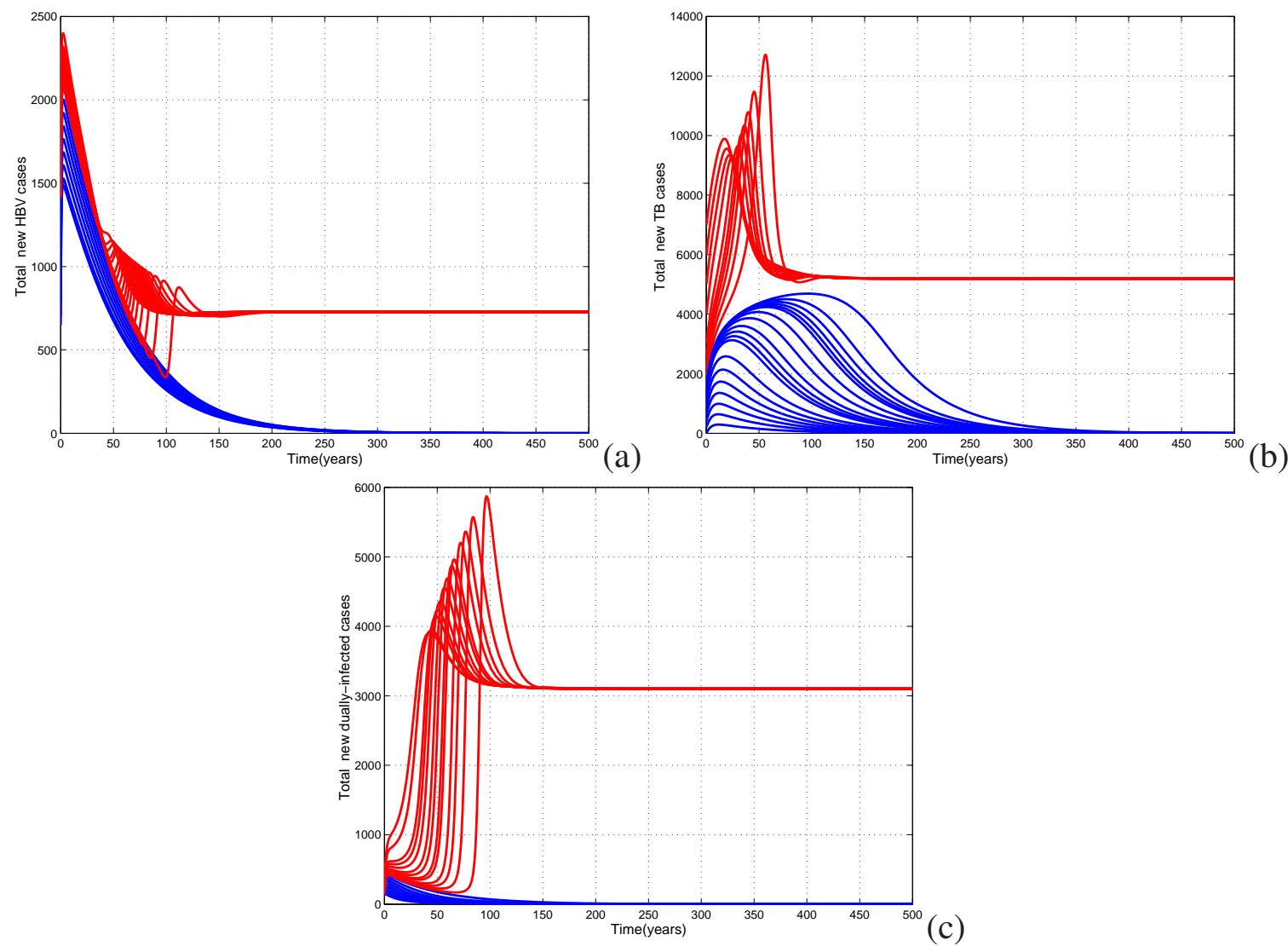

Figure 9: Simulations of the HBV-TB model (2.4) with different initial conditions illustrating the phenomenon of backward bifurcation when $\beta_{T}=1$ and $\beta_{H}=10$ (so that $\mathcal{R}_{0}^{H}=0.5805$, $\mathcal{R}_{0}^{T}=0.8733, \mathcal{R}_{H}^{T}=0.7363$ and $\left.\mathcal{R}_{0}=0.7363\right)$. This corresponds to the case $\mathcal{R}_{0}^{H}<\mathcal{R}_{H}^{T}<1$. All other parameters are as in Table 3.

Now, we choose $\beta_{T}=2$ and $\beta_{H}=10$ (so that $\mathcal{R}_{0}^{H}=0.5805$ and $\mathcal{R}_{H}^{T}=1.4725$. This corresponds to $\mathcal{R}_{0}^{H}<1$ and $\mathcal{R}_{H}^{T}>1$. Numerical results are depicted in Fig. 13. From this figure, it clearly appears that the HBV-free equilibrium is stable. This implies that when $\mathcal{R}_{H}^{T}>1$, then it is possible that HBV becomes prevalent even though it cannot persist in the absence of TB (as $\left.\mathcal{R}_{0}^{H}<1\right)$.

\subsubsection{Impact of $\mathrm{HBV}$ on the prevalence level of $\mathrm{TB}$ infection}

In many epidemiological models, the magnitude of the reproduction number is associated with the level of infection. The same is true in the model (2.4). That is, the basic reproduction numbers for TB and HBV infections, $\mathcal{R}_{H}^{T}$ and $\mathcal{R}_{0}^{H}$ (see Eq. (4.2)), are directly related to the infection levels of the respective diseases (in the absence of the other disease). Thus, we consider the impact of HBV on TB by first examining the effect of $k, k_{c}, k_{h}$ and $k_{r}$ on the prevalence of TB. After, we also examine the effect of $\sigma_{c}, \sigma_{h}$ and $\sigma_{r}$ on the prevalence of TB. The basic reproductive numbers 

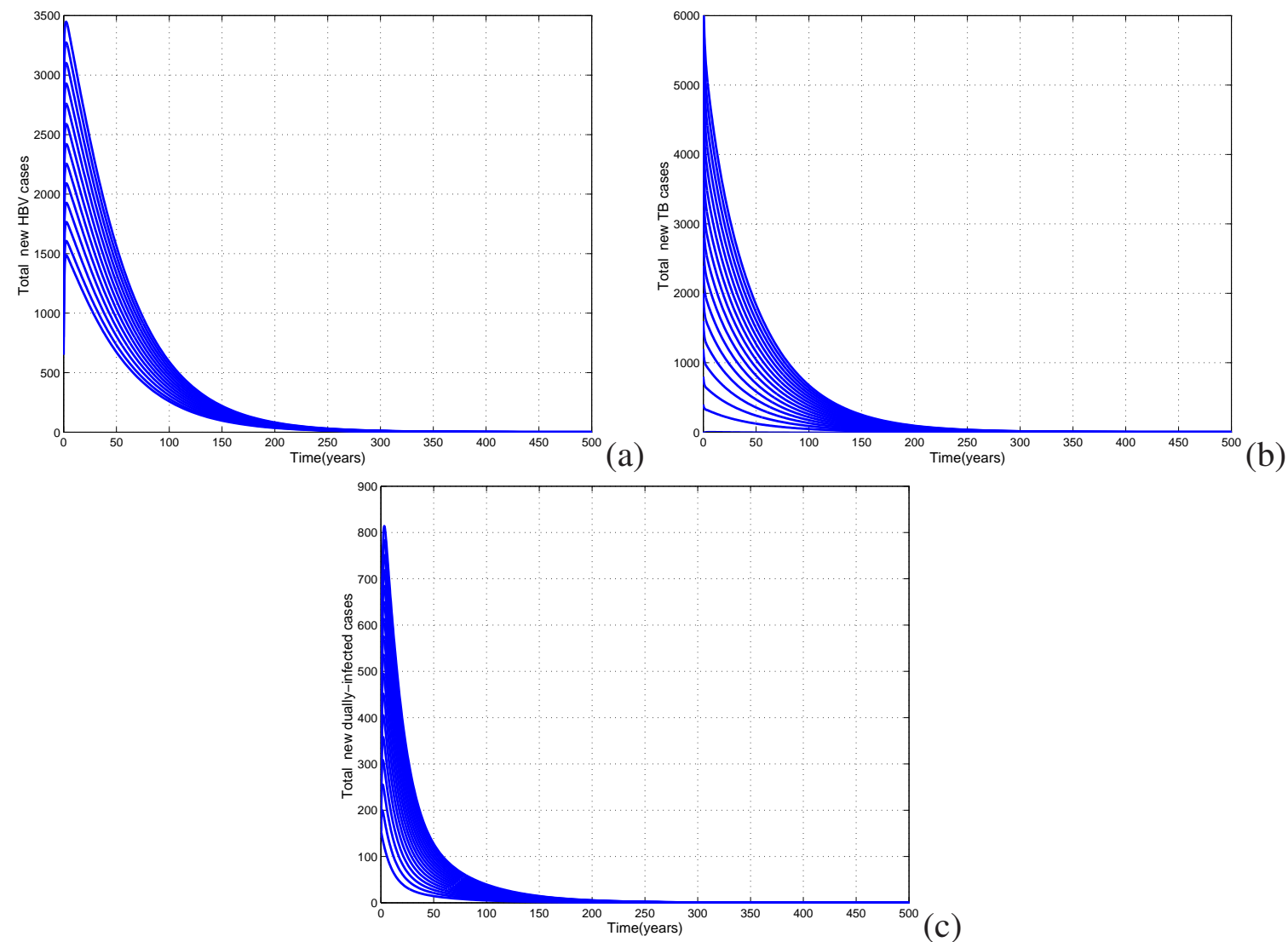

Figure 10: Simulations of the HBV-TB model (2.4) with different initial conditions when $\beta_{T}=$ 0.1 and $\beta_{H}=10$ (so that $\mathcal{R}_{0}^{H}=0.5805, \mathcal{R}_{0}^{T}=0.0873$ and $\mathcal{R}_{H}^{T}=0.0736$ ). This corresponds to the case where $\mathcal{R}_{H}^{T}<\mathcal{R}_{0}^{H}<1$. All other parameters are as in Table 3.

are the same for both plots: $\mathcal{R}_{0}^{H}=1.609\left(\beta_{H}=20\right)$ and $\mathcal{R}_{H}^{T}=1.4725\left(\beta_{T}=2\right)$. All other parameter values are given in Table 3 except that $r_{2 h}=r_{2 c}=r_{2 r}=0.5 r_{2}$ (i.e., it takes twice as long to treat a TB infected person with HBV than without). Figure 14 plots the fraction of active TB $\left(I_{T}+\varepsilon\left(I_{T R}+\varepsilon_{t} I_{T I}+\varepsilon_{c} I_{T C}\right) / N\right.$ and the fraction of HBV acute and chronic carriers $\left(I_{H}+\eta_{1}\left(E_{T I}+\eta_{1 c} I_{T I}\right)+\eta\left(C_{H}+\eta_{2}\left(E_{T C}+\eta_{2 c} I_{T C}\right)\right) / N\right.$.

Figures 14(a) and 14(b) present the results of the numerical simulation for different values of $k$, $k_{c}, k_{h}$ and $k_{r}$. It clearly appears that increase the parameters $k, k_{c}, k_{h}$ and $k_{r}$ due to TB has no effect on the prevalence levels of HBV and TB. This illustrates that the assumption $k_{c}>k_{h}>k_{r}>k$ does not automatically lead to an increase in TB prevalence. One of the reasons is that a person with both HBV acute and TB infection $\left(E_{T I}\right.$ and $\left.I_{T I}\right)$ may progress at a faster rate $\left(q_{1}>q_{2}\right)$ to the both HBV chronic and TB infection ( $E_{T C}$ and $I_{T C}$ ) which is associated with an excess death rate $\left(d_{2 T}, d_{4 T}>0\right)$. Figure 14(c) and 14(d) show the results for different values $\sigma_{c}, \sigma_{h}$ and $\sigma_{r}$. It shows that the increased the parameters $\sigma_{c}, \sigma_{h}$ and $\sigma_{r}$ due to TB have increased the prevalence level of TB but does not automatically lead to an increase in the HBV prevalence. In summary, conditions $k_{c}>k_{h}>k_{r}>k$ alone may not be sufficient for HBV to have a significant impact on TB. 

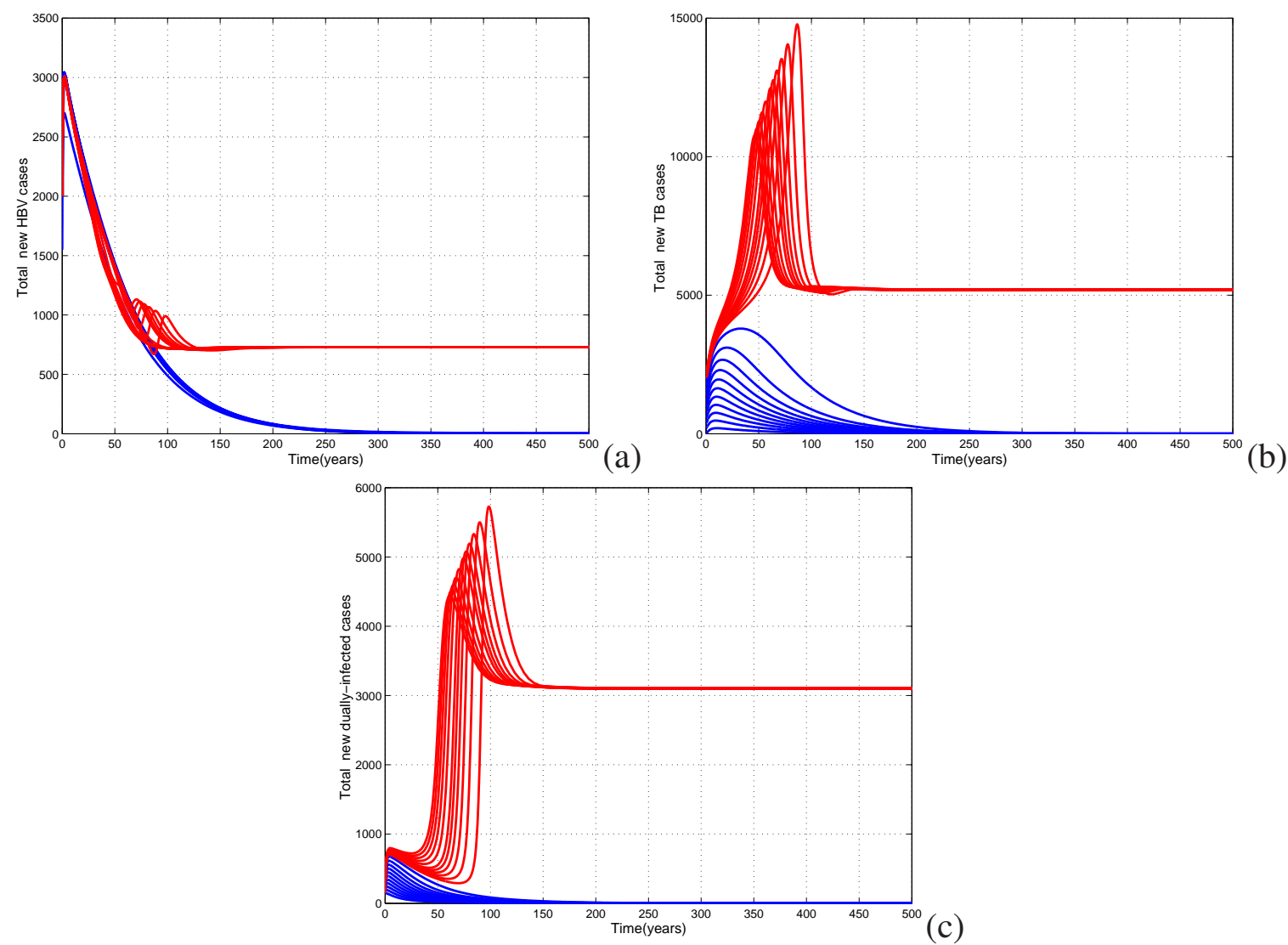

Figure 11: Time plots of trajectories of the HBV-TB model (2.4) with different initial conditions when $\beta_{T}=1$ and $\beta_{H}=20$ (so that $\left(\mathcal{R}_{0}^{H}=1.1609, \mathcal{R}_{0}^{T}=0.8733\right.$ and $\mathcal{R}_{H}^{T}=0.7363$ ). This corresponds to $\mathcal{R}_{0}^{H}>1$ and $\mathcal{R}_{H}^{T}<1$. All other parameters are as in Table 3 .

Other factors may also play an important role, e.g., the probability that individuals who are also infected with TB in the HBV acute infection fail to clear an acute state and develop a carrier state $\left(q_{1}\right.$ and $\left.q_{2}\right)$.

\subsubsection{Influence of TB on HBV dynamics}

Our numerical simulations also suggest that the presence of TB may have a significant impact on HBV dynamics. Simulation results are demonstrated in Fig. 14. This figure shows time plots of $\left(I_{T}+\varepsilon\left(I_{T R}+\varepsilon_{t} I_{T I}+\varepsilon_{c} I_{T C}\right) / N\right.$ (fraction of active TB) and $\left(I_{H}+\eta_{1}\left(E_{T I}+\eta_{1 c} I_{T I}\right)+\eta\left(C_{H}+\right.\right.$ $\left.\eta_{2}\left(E_{T C}+\eta_{2 c} I_{T C}\right)\right) / N$ ( fraction of HBV acute and chronic carriers) for different values of $q, q_{1}$ and $q_{2}$ (probability that an individual in the HBV acute infection fail to clear an acute state and develops a carrier state). Other parameter values are the same as in Fig. 14.

Figure 15(a) is for the case of $q=q_{1}=q_{2}$ (i.e., the probability that an individual in the HBV acute infection fail to clear an acute state and develops a carrier state is the same for a person with or without TB infection), while Fig. 15(b) is for the case of $q_{1}=10 q$ and $q_{2}=2 q$ (i.e., for HBV 

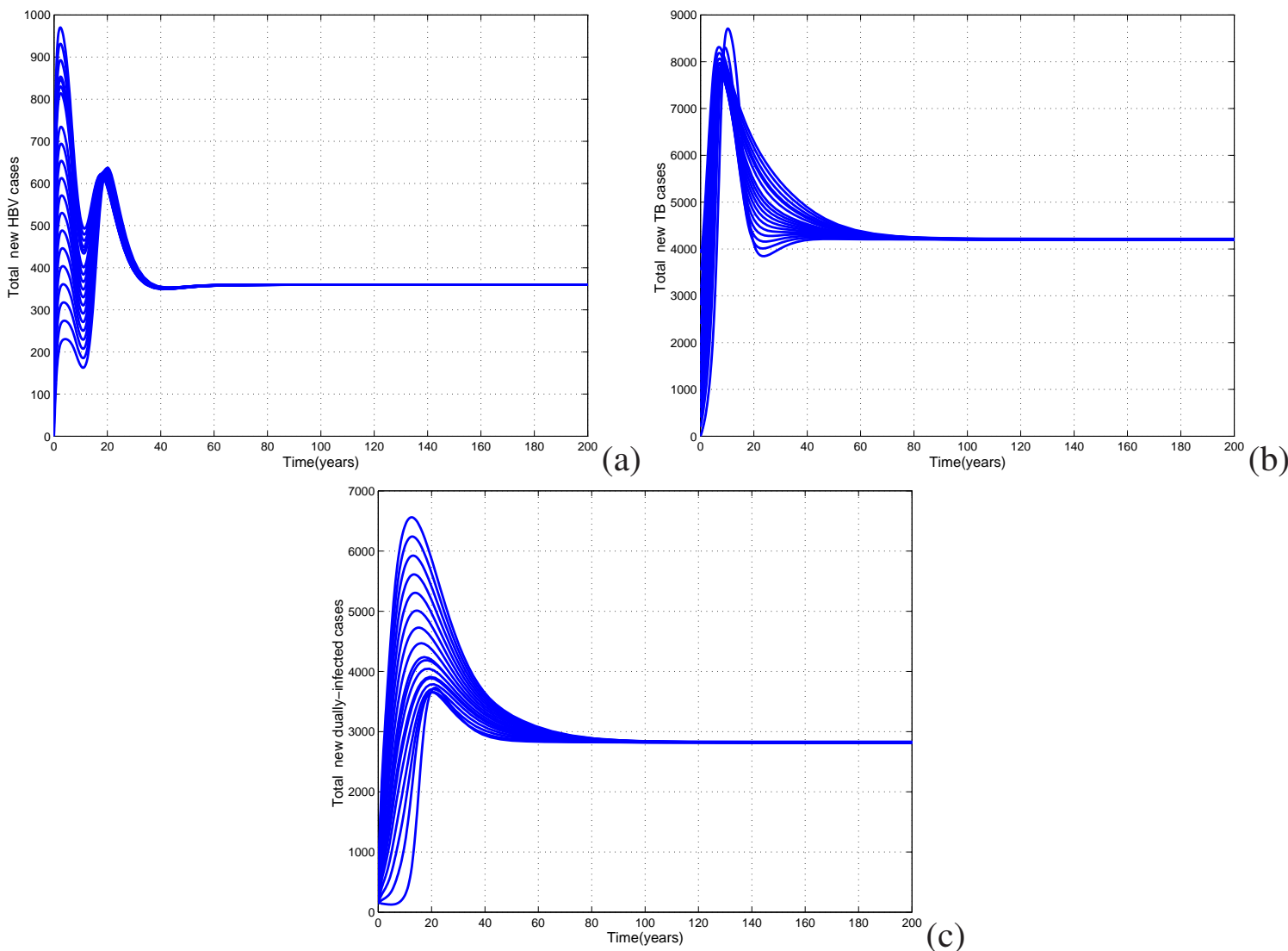

Figure 12: Time plots of trajectories of the HBV-TB model (2.4) with different initial conditions when $\beta_{T}=2$ and $\beta_{H}=20$ (so that $\mathcal{R}_{0}^{H}=1.1609, \mathcal{R}_{0}^{T}=1.7467$ and $\mathcal{R}_{H}^{T}=1.4725$ ). This corresponds to $\mathcal{R}_{0}^{H}>1$ and $\mathcal{R}_{H}^{T}>1$. All other parameters are as in Table 3 .

dually infected individuals with TB infection, the progression from acute stage to chronic stage of infection is respectively ten and two times faster than a person without TB infection). It shows that the increased the parameters $q_{1}$ and $q_{2}$ due to TB have increased the prevalence levels of HBV and TB. This means that condition $q_{1}>q_{2}>q$ alone may be sufficient for TB to have a significant impact on HBV.

\section{Discussions}

A realistic deterministic ODE based compartmental model for the transmission of hepatitis B and tuberculosis co-dynamics in a population has been proposed and analyzed. The HBV-only and TB-only models were qualitatively examined, first of all. The main theoretical results obtained are as follows:

1. We are able to compute independent reproduction numbers for $\mathrm{TB}\left(\mathcal{R}_{H}^{T}\right)$ and $\mathrm{HBV}\left(\mathcal{R}_{0}^{H}\right)$ and the total reproduction number for the system, $\mathcal{R}_{0}=\max \left\{\mathcal{R}_{H}^{T}, \mathcal{R}_{0}^{H}\right\}$. 

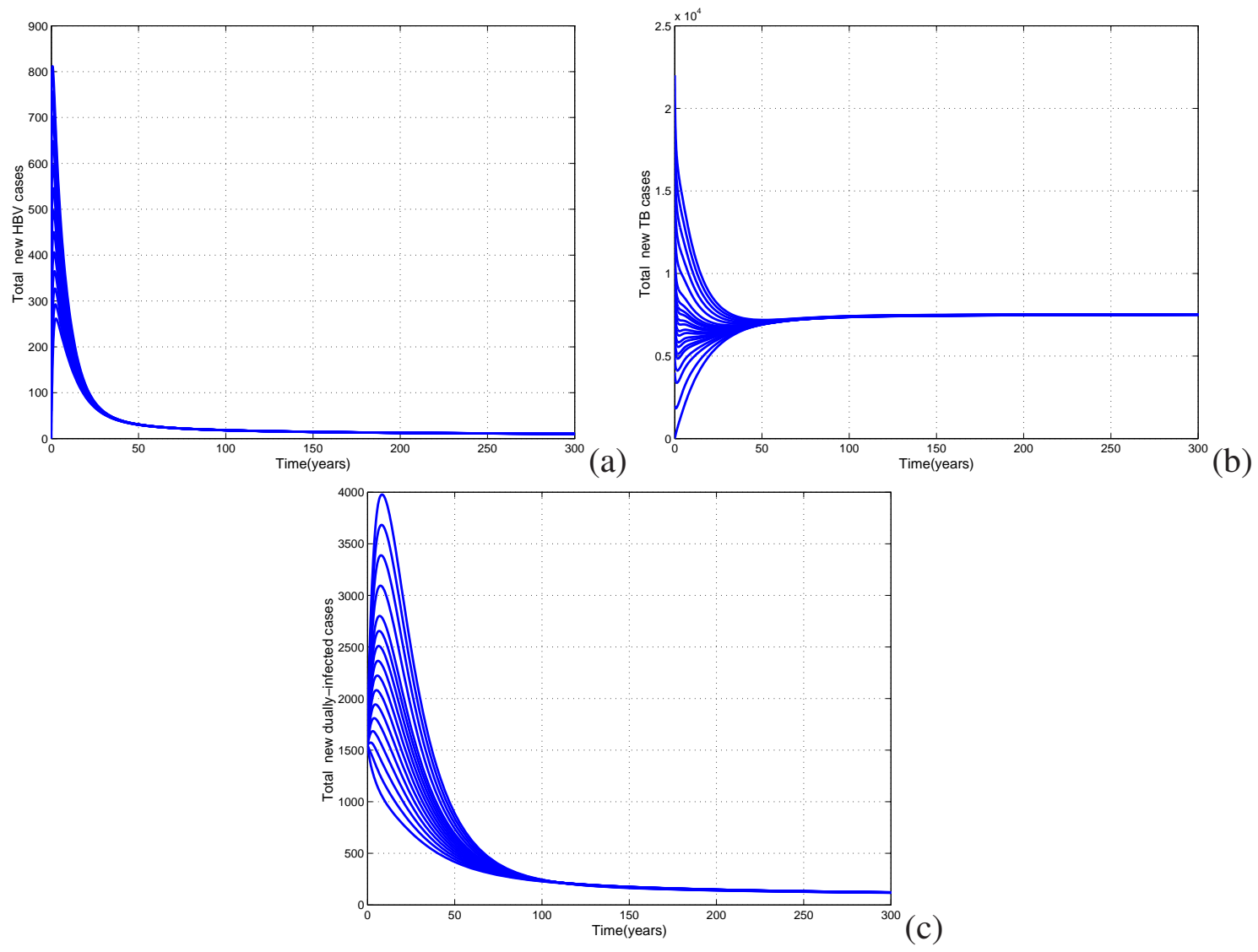

Figure 13: Simulations of the HBV-TB model (2.4) with different initial conditions when $\beta_{T}=$ 2 and $\beta_{H}=10$ (so that $\mathcal{R}_{0}^{H}=0.5805$ and $\mathcal{R}_{H}^{T}=1.4725$ ). This corresponds to $\mathcal{R}_{0}^{H}<1$ and $\mathcal{R}_{H}^{T}>1$. All other parameters are as in Table 3 .

2. The HBV-only model has a globally-asymptotically stable disease-free equilibrium whenever a certain epidemiological threshold $\left(\mathcal{R}_{0}^{H}\right)$ is less than unity and unstable if this threshold exceeds unity;

3. The HBV-only model has a unique endemic equilibrium whenever the aforementioned threshold exceeds unity. This endemic equilibrium is locally asymptotically stable at least near $\mathcal{R}_{0}^{H}=1$ whenever it exists;

4. Unlike the HBV-only model, the TB-only model undergoes the phenomenon of backward bifurcation, where the associated stable disease-free equilibrium co-exists with a stable endemic equilibrium when the corresponding reproduction number $\left(\mathcal{R}_{0}^{T}\right)$ is less than unity;

5. The full HBV-TB model is shown to have a locally-asymptotically stable disease-free equilibrium when its reproductive threshold is less than unity, and unstable if the threshold exceeds unity. It also undergoes the phenomenon of backward bifurcation under certain conditions; 

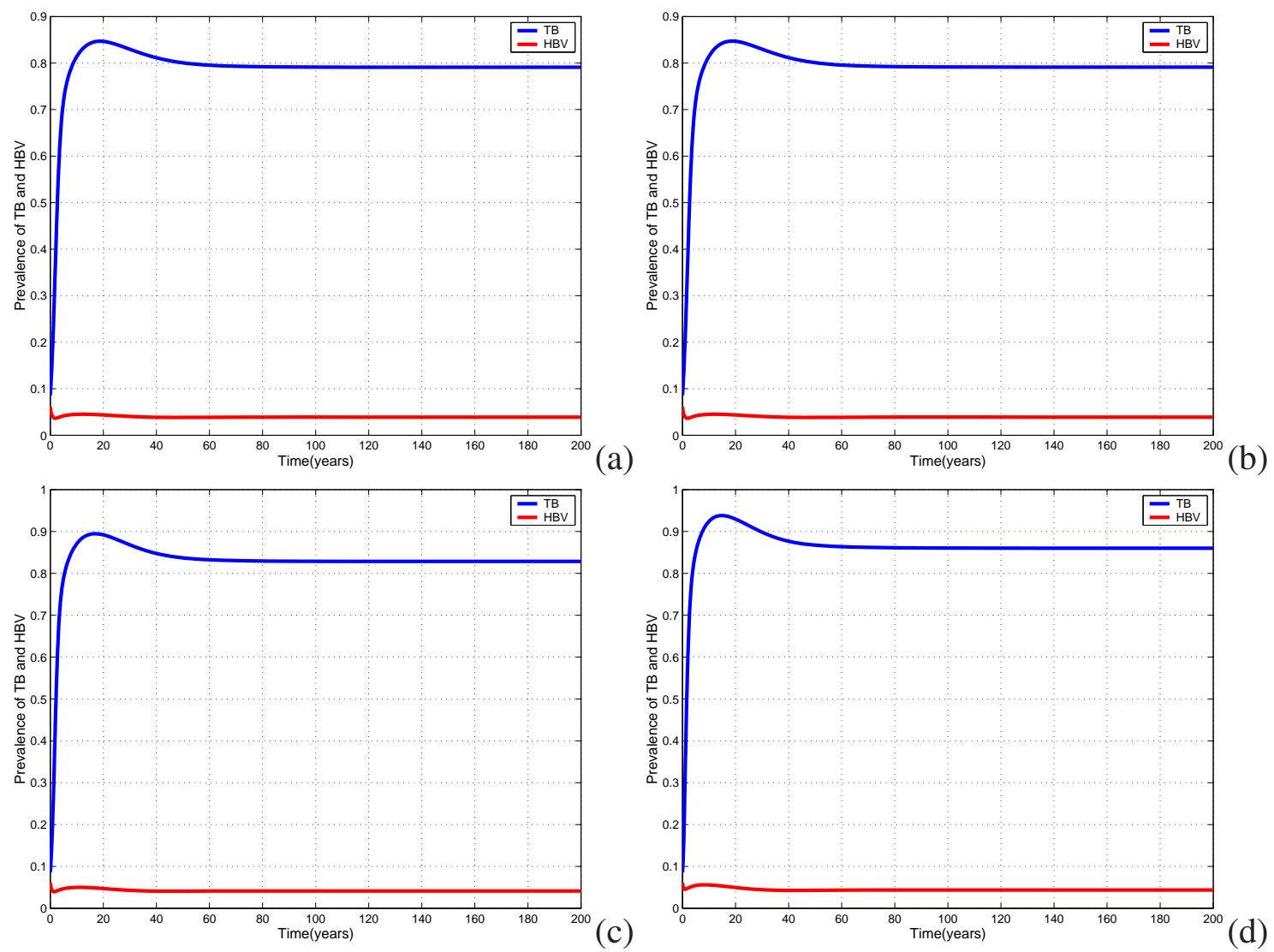

Figure 14: Time plots of fraction of active TB and fraction of HBV acute and chronic carriers when $\beta_{T}=2$ and $\beta_{H}=20$ (so that $\mathcal{R}_{0}^{H}=1.609$ and $\mathcal{R}_{H}^{T}=1.4725$ ) and $r_{2 h}=r_{2 c}=r_{2 r}=$ $0.5 r_{2}$. (i) For different values of $k, k_{h}, k_{c}$ and $k_{r}$ : (a) $k=k_{h}=k_{c}=k_{r}$ and (b) $k_{c}=5 k$, $k_{h}=3 k$ and $k_{r}=2 k$; and (ii) for different values of $\sigma, \sigma_{h}, \sigma_{c}$ and $\sigma_{r}$ (c) $\sigma=\sigma_{h}=\sigma_{c}=\sigma_{r}$ and (d) $\sigma_{c}=5 \sigma, \sigma_{h}=3 \sigma$ and $\sigma_{r}=2 \sigma$. All other parameter values are given in Table 3 except that $r_{2 h}=r_{2 c}=r_{2 r}=0.5 r_{2}$ (i.e., it takes twice as long to treat a TB infected person with HBV than without).

The simulation results provided many interesting insights into the effect of the dynamical interactions between TB and HBV. Mainly, the following are observed:

(i) The HBV only sub-model has a DFE which is GAS.

(ii) The analysis shows that the TB-only model exhibits a mechanism known as backward bifurcation by allowing for reactivation of latent TB individuals by infected individuals.

(iii) The two diseases disappear in the population whenever their reproduction numbers are very small and less than unity.

(iv) The HBV-free equilibrium is stable when $\mathcal{R}_{H}^{T}>1$ and $\mathcal{R}_{0}^{H}<1$. However, the symmetric result does not hold. That is, the TB-free equilibrium may not be stable when $\mathcal{R}_{H}^{T}<1$ and $\mathcal{R}_{0}^{H}>1$.

(v) The two diseases co-exist whenever their reproduction numbers exceed unity (regardless 

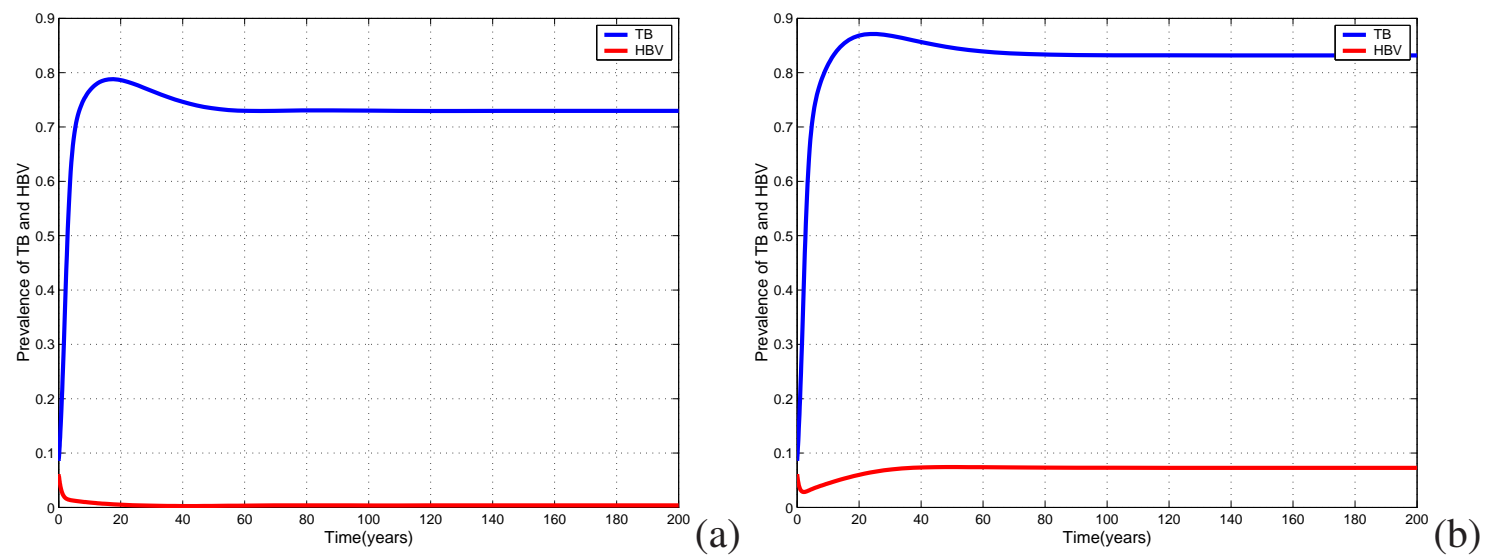

Figure 15: Time plots of fraction of active TB and fraction of HBV acute and chronic carriers for different values of $q, q_{1}$ and $q_{2}$. (a) $q=q_{1}=q_{2}$, and (b) $q_{1}=10 q$ and $q_{2}=2 q$. Other parameter values are the same as in Fig. 14.

which of the numbers is larger).

(vi) Condition $k_{c}>k_{h}>k_{r}>k$ alone does not always lead to a significant increase in TB in the presence of HBV. However, when $\sigma_{c}>\sigma_{h}>\sigma_{r}>\sigma$, the presence of HBV may have a significant influence on TB dynamics. Also, when $q_{1}>q_{2}>q$, the presence of TB may have a significant influence on the HBV dynamics.

Numerical results suggest that to reduce or control the impact of TB, investing more in reducing the prevalence of HBV can be an effective option. Such reductions would not be easy due to the waning vaccine-induced immunity and lack of effective medication. There is no 'cure' at this time for HBV. However, significant reductions may be obtained through programs that accelerate the treatment of active TB cases. Since there are about 8 million new cases of active TB per year, a program may be feasible. It has worked well in countries that allocate substantial resources to public health. Naturally, accelerating the treatment rate of individuals with active TB is more critical in areas where HIV prevalence is high since approximately $10 \%$ of the HIV-infected population worldwide is infected with HBV. Unfortunately, the areas with the highest prevalence of co-infections have limited resources and cannot implement to accelerated TB treatment programs.

It is worth stressing that our models and observations are rather crude for multiple reasons. The results are not only based on local mathematical analysis but they are from a model that does not incorporate multiple and common forms of the possibility of increases in antibiotic resistant $\mathrm{TB}$, and the impact of human behavior, particularly the role of core groups (prostitution, injection drug, etc.). A research program that tackles some of these possibilities is still viable, since it is the first time the dynamics of HBV-TB co-infection is studied and therefore they are still poorly understood from a theoretical or mathematical perspective. 


\section{Acknowledgements}

The authors gratefully acknowledges the support of the Alexander von Humboldt Foundation, Germany. We would like to thank the anonymous referee for his/her careful reading and helpful suggestions which led to an improvement of our original manuscript.

\section{Appendix A: Local stability of the unique endemic equilibrium of the HBV-only model (3.1)}

In this Appendix, we shall give the proof of Lemma 4, using the Centre Manifold theory. To apply this theory, the following simplification and change of variables are made first of all. Let $x_{1}=S, x_{2}=V, x_{3}=I_{H}, x_{4}=C_{H}$ and $x_{5}=R_{H}$ so that $N=x_{1}+x_{2}+x_{3}+x_{4}+x_{5}$. Further, by using vector notation $x=\left(x_{1}, x_{2}, x_{3}, x_{4}, x_{5}\right)^{T}$, the HBV-only model (3.1) can be written in the form $\dot{x}=f(x)$, with $f=\left(f_{1}, f_{2}, f_{3}, f_{4}, f_{5}\right)^{T}$, as follows:

$$
\left\{\begin{array}{l}
\dot{x}_{1}=f_{1}=\Lambda+\mu \omega\left(1-v x_{4}\right)-(1-\delta) \lambda_{H} x_{1}-\left(\delta+\mu_{0}\right) x_{1}, \\
\dot{x}_{2}=f_{2}=\mu(1-\omega)+\delta x_{1}-\pi_{H} \lambda_{H} x_{2}-\mu_{0} x_{2}, \\
\dot{x}_{3}=f_{3}=\left[\pi_{H} x_{2}+(1-\delta) x_{1}\right] \lambda_{H}-\left(\mu_{0}+\gamma_{1}\right) x_{3}, \\
\dot{x}_{4}=f_{4}=\mu \omega v x_{4}+q \gamma_{1} x_{3}-\left(\mu_{0}+d_{H}+\gamma_{2}\right) x_{4}, \\
\dot{x}_{5}=f_{5}=\gamma_{1}(1-q) x_{3}+\gamma_{2} x_{4}-\mu_{0} x_{5},
\end{array}\right.
$$

where

$$
\lambda_{H}=\frac{\beta_{H}\left(x_{3}+\eta x_{4}\right)}{x_{1}+x_{2}+x_{3}+x_{4}+x_{5}} .
$$

The Jacobian of system (5.1), at the DFE $Q_{0}^{H}$, is given by

$$
J\left(Q_{0}^{H}\right)=\left(\begin{array}{ccccc}
-\left(\mu_{0}+\delta\right) & 0 & -J_{1} & -J_{2} & 0 \\
\delta & -\mu_{0} & -J_{3} & -J_{4} & 0 \\
0 & 0 & J_{5} & J_{6} & 0 \\
0 & 0 & q \gamma_{1} & -A & 0 \\
0 & 0 & \gamma_{1}(1-q) & \gamma_{2} & -\mu_{0}
\end{array}\right)
$$

where

$$
\begin{aligned}
J_{1} & =\frac{\beta_{H}(1-\delta) x_{01}}{x_{01}+x_{02}}, \quad J_{2}=\mu \omega v+\frac{\beta_{H} \eta(1-\delta) x_{01}}{x_{01}+x_{02}}, \quad J_{3}=\frac{\beta_{H} \pi_{H} x_{02}}{x_{01}+x_{02}}, \\
J_{4} & =\frac{\beta_{H} \pi_{H} \eta x_{02}}{x_{01}+x_{02}}, \quad J_{5}=\frac{\beta_{H}\left[\pi_{H} x_{02}+(1-\delta) x_{01}\right]}{x_{01}+x_{02}}-\mu_{0}-\gamma_{1} \\
J_{6} & =\frac{\beta_{H} \eta\left[\pi_{H} x_{02}+(1-\delta) x_{01}\right]}{x_{01}+x_{02}},
\end{aligned}
$$


with

$$
x_{01}=\frac{\Lambda+\mu \omega}{\mu_{0}+\delta} \quad \text { and } \quad x_{02}=\frac{\delta(\mu+\Lambda)+\mu \mu_{0}(1-\omega)}{\mu_{0}\left(\mu_{0}+\delta\right)} .
$$

Consider, next, the case when $\mathcal{R}_{0}^{H}=1$. Suppose, further, that $\beta_{H}=\beta_{H}^{*}$ is chosen as a bifurcation parameter. Solving for $\beta_{H}$ from $\mathcal{R}_{0}^{H}=1$ gives

$$
\beta_{H}=\frac{A\left(\mu_{0}+\delta\right)\left(\mu_{0}+\gamma_{1}\right)(\Lambda+\mu)}{\left(A+\eta q \gamma_{1}\right)\left[\pi_{H}\left[\delta(\mu+\Lambda)+\mu \mu_{0}(1-\omega)\right]+\mu_{0}(1-\delta)(\Lambda+\mu \omega)\right]} .
$$

It follows that the Jacobian $\left(J\left(Q_{0}^{H}\right)\right)$ of $(5.1)$ at the DFE, with $\beta_{H}=\beta_{H}^{*}$, denoted by $J_{\beta_{H}^{*}}$ has a simple zero eigenvalue (with all other eigenvalues having negative real part). Hence, the Centre Manifold theory [56] can be used to analyze the dynamics of the model (5.1). In particular, the theorem in [57] (see also [52, 56, 58]), reproduced below for convenience, will be used to show that the unique endemic equilibrium of the model (5.1) (or, equivalently, (3.1)) is locally asymptotically stable for $\mathcal{R}_{0}^{H}$ near 1 .

Theorem 8. (Castillo-Chavez \& Song [57]). Consider the following general system of ordinary differential equations with a parameter $\phi$ :

$$
\frac{d x}{d t}=f(x, \phi), \quad f: \mathbb{R}^{n} \times \mathbb{R} \rightarrow \mathbb{R} \quad \text { and } \quad f \in C^{2}\left(\mathbb{R}^{n}, \mathbb{R}\right),
$$

where 0 is an equilibrium point of the system (that is, $f(0, \phi) \equiv 0$ for all $\phi$ ) and assume

1. $A=D_{x} f(0,0)=\left(\frac{\partial f_{i}}{\partial x_{j}}(0,0)\right)$ is the linearization matrix of system (5.1) around the equilibrium 0 with $\phi$ evaluated at 0 . Zero is a simple eigenvalue of $A$ and other eigenvalues of $A$ have negative real parts;

2. Matrix A has a right eigenvector $u$ and a left eigenvector $z$ (each corresponding to the zero eigenvalue).

Let $f_{k}$ be the $k^{\text {th }}$ component of $f$ and

$$
\begin{aligned}
a & =\sum_{k, i, j=1}^{n} z_{k} u_{i} u_{j} \frac{\partial^{2} f_{k}}{\partial x_{i} \partial x_{j}}(0,0), \\
b & =\sum_{k, i=1}^{n} z_{k} u_{i} \frac{\partial^{2} f_{k}}{\partial x_{i} \partial \phi}(0,0),
\end{aligned}
$$

then the local dynamics of the system around the equilibrium point 0 is totally determined by the signs of $a$ and $b$.

1. $a>0, b>0$. When $\phi<0$ with $|\phi| \ll 1,0$ is locally asymptotically stable and there exists a positive unstable equilibrium; when $0<\phi \ll 0,0$ is unstable and there exists a negative, locally asymptotically stable equilibrium; 
2. $a<0, b<0$. When $\phi<0$ with $|\phi| \ll 1$, 0 is unstable; when $0<\phi \ll 1$, 0 is locally asymptotically stable equilibrium, and there exists a positive unstable equilibrium;

3. $a>0, b<0$. When $\phi<0$ with $|\phi| \ll 1,0$ is unstable, and there exists a locally asymptotically stable negative equilibrium; when $0<\phi \ll 1,0$ is stable, and a positive unstable equilibrium appears;

4. $a<0, b>0$. When $\phi$ changes from negative to positive, 0 changes its stability from stable to unstable. Correspondingly a negative unstable equilibrium becomes positive and locally asymptotically stable.

Particularly, if $a>0$ and $b>0$, then a backward bifurcation occurs at $\phi=0$.

In order to apply the above theorem, the following computations are necessary (it should be noted that we are using $\beta_{H}^{*}$ as the bifurcation parameter, in place of $\phi$ in Theorem 8).

Eigenvectors of $J_{\beta_{H}^{*}}$ : For the case when $\mathcal{R}_{0}^{H}=1$, it can be shown that the Jacobian of (5.1) at $\beta_{H}=\beta_{H}^{*}$ (denoted by $J_{\beta_{H}^{*}}$ ) has a right eigenvector (corresponding to the zero eigenvalue), given by $u=\left(u_{1}, u_{2}, u_{3}, u_{4}, u_{5}\right)^{T}$, where,

$$
\left\{\begin{array}{l}
u_{1}=-\frac{\left(A J_{1}+q \gamma_{1} J_{2}\right) u_{3}}{A\left(\mu_{0}+\delta\right)}, \\
u_{2}=-\frac{\left[\delta\left(A J_{1}+q \gamma_{1} J_{2}\right)+\left(\mu_{0}+\delta\right)\left(A J_{3}+q \gamma_{1} J_{4}\right)\right] u_{3}}{A \mu_{0}\left(\mu_{0}+\delta\right)}, \\
u_{3}=u_{3}>0, \\
u_{4}=\frac{q \gamma_{1}}{A} u_{3}, \\
u_{5}=\frac{\gamma_{1}}{\mu_{0}}\left(1-q+\frac{q \gamma_{2}}{A}\right) u_{3} .
\end{array}\right.
$$

Similarly, the components of the left eigenvectors of $J_{\beta^{*}}$ (corresponding to the zero eigenvalue), denoted by $z=\left(z_{1}, z_{2}, z_{3}, z_{4}, z_{5}\right)^{T}$, are given by,

$$
\left\{\begin{aligned}
z_{1} & =0 \\
z_{2} & =0 \\
z_{3} & =z_{3}>0 \\
z_{4} & =\frac{J_{6}}{A} z_{3} \\
z_{5} & =0
\end{aligned}\right.
$$


Computation of $a$ : For system (5.1), the associated non-zero partial derivatives of $f$ (at the DFE) are given by

$$
\begin{aligned}
& \frac{\partial^{2} f_{3}}{\partial x_{3}^{2}}=-\frac{2 \beta_{H}^{*}\left[\pi_{H} x_{02}+(1-\delta) x_{01}\right]}{\left(x_{01}+x_{02}\right)^{2}}, \\
& \frac{\partial^{2} f_{3}}{\partial x_{3} \partial x_{4}}=\frac{\partial^{2} f_{3}}{\partial x_{4} \partial x_{3}}=-\frac{\beta_{H}^{*}(1+\eta)\left[\pi_{H} x_{02}+(1-\delta) x_{01}\right]}{\left(x_{01}+x_{02}\right)^{2}}, \\
& \frac{\partial^{2} f_{3}}{\partial x_{1} \partial x_{3}}=\frac{\partial^{2} f_{3}}{\partial x_{3} \partial x_{1}}=-\frac{\beta_{H}^{*}\left(\pi_{H}+\delta-1\right) x_{02}}{\left(x_{01}+x_{02}\right)^{2}}, \quad \frac{\partial^{2} f_{3}}{\partial x_{2} \partial x_{3}}=\frac{\partial^{2} f_{3}}{\partial x_{3} \partial x_{2}}=\frac{\beta_{H}^{*}\left(\pi_{H}+\delta-1\right) x_{01}}{\left(x_{01}+x_{02}\right)^{2}}, \\
& \frac{\partial^{2} f_{3}}{\partial x_{1} \partial x_{4}}=\frac{\partial^{2} f_{3}}{\partial x_{4} \partial x_{1}}=-\frac{\beta_{H}^{*} \eta\left(\pi_{H}+\delta-1\right) x_{02}}{\left(x_{01}+x_{02}\right)^{2}}, \quad \frac{\partial^{2} f_{3}}{\partial x_{2} \partial x_{4}}=\frac{\partial^{2} f_{3}}{\partial x_{4} \partial x_{2}}=\frac{\beta_{H}^{*} \eta\left(\pi_{H}+\delta-1\right) x_{01}}{\left(x_{01}+x_{02}\right)^{2}}, \\
& \frac{\partial^{2} f_{3}}{\partial x_{4}^{2}}=-\frac{2 \beta_{H}^{*} \eta\left[\pi_{H} x_{02}+(1-\delta) x_{01}\right]}{\left(x_{01}+x_{02}\right)^{2}}=\frac{\partial^{2} f_{3}}{\partial x_{3} \partial x_{5}}=-\frac{\beta_{H}^{*}\left[\pi_{H} x_{02}+(1-\delta) x_{01}\right]}{\left(x_{01}+x_{02}\right)^{2}}, \\
& \frac{\partial^{2} f_{3}}{\partial x_{5} \partial x_{4}}=\frac{\partial^{2} f_{3}}{\partial x_{4} \partial x_{5}}=-\frac{\beta_{H}^{*} \eta\left[\pi_{H} x_{02}+(1-\delta) x_{01}\right]}{\left(x_{01}+x_{02}\right)^{2}} .
\end{aligned}
$$

Then, it follows that

$$
\begin{aligned}
a & =z_{3} \sum_{i, j=1}^{5} u_{i} u_{j} \frac{\partial^{2} f_{3}}{\partial x_{i} \partial x_{j}}, \\
& =-\frac{2 \beta_{H}^{*} z_{3}\left(u_{3}+\eta u_{4}\right)}{\left(x_{01}+x_{02}\right)^{2}}\left[\left[\pi_{H} x_{02}+(1-\delta) x_{01}\right]\left(u_{3}+u_{4}+u_{5}\right)+\left(\pi_{H}+\delta-1\right)\left(-x_{01} u_{2}+x_{02} u_{1}\right)\right] .
\end{aligned}
$$

Now, using Eq. (5.3) and after a calculation, one finally obtains

$$
a=-\frac{2 \beta_{H}^{*} z_{3} u_{3}\left(u_{3}+\eta u_{4}\right)}{A \mu_{0}\left(\mu_{0}+\delta\right)\left(x_{01}+x_{02}\right)^{2}}\left[\mathcal{A}_{1}+\pi_{H} \mathcal{A}_{2}+(1-\delta) \mathcal{A}_{3}\right]
$$

where

$$
\begin{aligned}
\mathcal{A}_{1} & =\frac{\beta_{H} \mu_{0}\left(A+q \gamma_{1} \eta\right)\left(\pi_{H}+\delta-1\right)^{2} x_{01} x_{02}}{N_{0}} \\
\mathcal{A}_{2} & =\frac{\beta_{H} \delta\left(A+q \gamma_{1} \eta\right)\left[\pi_{H} x_{02}+(1-\delta) x_{01}\right] x_{01}}{N_{0}}+x_{02}\left(\mu_{0}+\delta\right)\left[\mu_{0}+\gamma_{1}(1-q)\right] A, \\
& +\frac{q \gamma_{1}}{\mu_{0}}\left[\mu \mu_{0}(1-\omega)\left(\mu_{0}+\gamma_{2}-\mu \omega v\right)+\delta(\Lambda+\mu)\left(\mu_{0}+\gamma_{2}\right)\right], \\
\mathcal{A}_{3} & =q \gamma_{1} \mu^{2} \omega v(1-\omega)+x_{01} q \gamma_{1}\left(\mu_{0}+\gamma_{2}\right)+x_{01} A\left[\mu_{0}+\gamma_{1}+\mu_{0}\left(\mu_{0}+\delta\right)+\gamma_{1}(1-q)\left(\mu_{0}+\delta\right)\right] .
\end{aligned}
$$


At this stage, remind that we have assumed that $\mu \omega v<\mu_{0}+d_{H}+\gamma_{2}$ because if this inequality do not hold, $d C_{H} / d t>0$ for $C_{H} \neq 0$ or $I_{H}>0$ and $t \geq 0$. Note also that when $\mu \omega v<\mu_{0}+\gamma_{2}$, carriers would also not keep increasing rapidly as long as there is infection. Then, we can also assumed that $\mu_{0}+\gamma_{2}-\mu \omega v>0$. In this case, one has $a<0$.

Computation of $\mathbf{b}$ : For the sign of $b$, it can be shown that the associated non-vanishing partial derivatives of $f$ are

$$
\frac{\partial^{2} f_{3}}{\partial x_{3} \partial \beta_{H}^{*}}(0,0)=\frac{\pi_{H} x_{02}+(1-\delta) x_{01}}{x_{01}+x_{02}} \quad \text { and } \quad \frac{\partial^{2} f_{3}}{\partial x_{4} \partial \beta_{H}^{*}}(0,0)=\frac{\eta\left[\pi_{H} x_{02}+(1-\delta) x_{01}\right]}{x_{01}+x_{02}}
$$

Substituting the respective partial derivatives into the expression

$$
b=z_{3} \sum_{i=1}^{5} u_{i} \frac{\partial^{2} f_{3}}{\partial x_{i} \beta_{H}^{*}}(0,0)+z_{4} \sum_{i=1}^{5} u_{i} \frac{\partial^{2} f_{4}}{\partial x_{i} \beta_{H}^{*}}(0,0) .
$$

gives

$$
b=\frac{z_{3}\left(u_{3}+\eta u_{4}\right)}{x_{01}+x_{02}}\left[\pi_{H} x_{02}+(1-\delta) x_{01}\right]>0 .
$$

Thus, $a<0$ and $b>0$. So (by Theorem 8, Item (4)), we have established the result about the local stability of the endemic equilibrium of the HBV-only model (note that this result holds for $\mathcal{R}_{0}^{H}>1$ but close to 1$)$. This achieves the proof.

\section{Appendix B: Calculation of the basic reproduction ratio for sys- tem (2.4)}

In order to compute the basic reproduction ratio, it is important to distinguish new infections from all other class transitions in the population. The infected classes are $I_{H}, C_{H}, R_{H}, E_{T}, I_{T}, I_{T I}$, $E_{T I}, I_{T C}, E_{T C}, I_{T R}$ and $E_{T R}$. Following Van den Driessche and Watmough [52], we can write the model (2.4) as

$$
\dot{x}=f(x)=\mathcal{F}(x)-\mathcal{V}(x)=\mathcal{F}(x)-\left(\mathcal{V}^{-}(x)-\mathcal{V}^{+}(x)\right),
$$

where $x=\left(I_{H}, C_{H}, R_{H}, E_{T}, I_{T}, E_{T I}, I_{T I}, E_{T C}, I_{T C}, E_{T R}, I_{T R}, S, V\right), \mathcal{F}$ is the rate of appearance of new infections in each class, $\mathcal{V}^{+}$is the rate of transfer into each class by all other means and $\mathcal{V}^{-}$ is the rate of transfer out of each class. Hence,

$$
\begin{gathered}
\mathcal{F}(x)=\left(\left[\pi_{H} V+(1-\delta) S\right] \lambda_{H} S, 0,0,[(1-f) V+(1-\delta)(1-p) S] \lambda_{T},\right. \\
\left.[f V+p(1-\delta) S] \lambda_{T}, 0,0,0,0,0,0,0,0\right)^{T} .
\end{gathered}
$$

The Jacobian matrices of $\mathcal{F}$ and $\mathcal{V}$ at the disease-free equilibrium $Q_{0}$ are

$$
D \mathcal{F}\left(Q_{0}\right)=\left[\begin{array}{cc}
F & 0 \\
0 & 0
\end{array}\right], \quad D \mathcal{V}\left(Q_{0}\right)=\left[\begin{array}{cc}
V & 0 \\
J_{3} & J_{4}
\end{array}\right]
$$


where $F$ and $V$ correspond to the derivatives of $\mathcal{F}$ and $\mathcal{V}$ with respect to the infected classes:

$$
F=\left[\begin{array}{ccc}
F_{H} & 0 & F_{T H} \\
0 & F_{T} & \tilde{F}_{T H} \\
0 & 0 & 0
\end{array}\right] \quad \text { and } \quad V=\left[\begin{array}{ccc}
V_{H} & 0 & 0 \\
0 & V_{T} & 0 \\
0 & 0 & V_{T H}
\end{array}\right]
$$

where

$$
\begin{aligned}
& F_{H}=\frac{\beta_{H}\left[\pi_{H} V_{0}+(1-\delta) S_{0}\right]}{S_{0}+V_{0}}\left[\begin{array}{ccc}
1 & \eta & 0 \\
0 & 0 & 0 \\
0 & 0 & 0
\end{array}\right], \quad F_{T}=\frac{\beta_{T}}{S_{0}+V_{0}}\left[\begin{array}{cc}
0 & F_{01} \\
0 & F_{02}
\end{array}\right], \\
& F_{T H}=\frac{\beta_{H}\left[\pi_{H} V_{0}+(1-\delta) S_{0}\right]}{S_{0}+V_{0}}\left[\begin{array}{cccccc}
\eta_{1} & \eta_{2} & \eta_{1} \eta_{1 c} & \eta_{2} \eta_{2 c} & 0 & 0 \\
0 & 0 & 0 & 0 & 0 & 0 \\
0 & 0 & 0 & 0 & 0 & 0
\end{array}\right] \\
& \tilde{F}_{T H}=\frac{\beta_{T}}{S_{0}+V_{0}}\left[\begin{array}{ccccccc}
0 & \varepsilon \varepsilon_{1} F_{01} & 0 & \varepsilon \varepsilon_{2} F_{01} & 0 & \varepsilon F_{01} \\
0 & \varepsilon \varepsilon_{1} F_{02} & 0 & \varepsilon \varepsilon_{2} F_{02} & 0 & \varepsilon F_{02}
\end{array}\right],
\end{aligned}
$$

and

$$
V_{H}=\left[\begin{array}{ccc}
\mu_{0}+\gamma_{1} & 0 & 0 \\
-q \gamma_{1} & A & 0 \\
-\gamma_{1}(1-q) & -\gamma_{2} & \mu_{0}
\end{array}\right], \quad V_{T}=\left[\begin{array}{cc}
A_{1} & -r_{2} \\
-k\left(1-r_{1}\right) & A_{2}
\end{array}\right]
$$

$V_{T H}=$

$$
\left[\begin{array}{cccccc}
v_{1 T} & -r_{2 h} & 0 & 0 & 0 & 0 \\
-k_{h}\left(1-r_{1 h}\right) & v_{2 T} & 0 & 0 & 0 & 0 \\
-q_{2} \phi_{2} & 0 & v_{3 T} & -r_{2 c} & 0 & 0 \\
0 & -q_{1} \phi_{1}\left(1-r_{2 h}\right) & -k_{c}\left(1-r_{1 c}\right) & v_{4 T} & 0 & 0 \\
-\phi_{2}\left(1-q_{2}\right) & 0 & -\theta_{2} & 0 & v_{5 T} & -r_{2 c} \\
0 & -\phi_{1}\left(1-q_{1}\right)\left(1-r_{2 h}\right) & 0 & -\theta_{1}\left(1-r_{2 c}\right) & -k_{r}\left(1-r_{1 r}\right) & v_{6 T}
\end{array}\right],
$$

with

$$
\begin{aligned}
& F_{01}=(1-f) V_{0}+(1-p)(1-\delta) S_{0}, \quad F_{02}=f V_{0}+p(1-\delta) S_{0}, \\
& v_{1 T}=\mu_{0}+k_{h}\left(1-r_{1 h}\right)+\phi_{2}, \quad v_{2 T}=\mu_{0}+d_{1 T}+r_{2 h}+\phi_{1}\left(1-r_{2 h}\right), \\
& v_{3 T}=\mu_{0}+d_{4 T}+\theta_{2}+k_{c}\left(1-r_{1 c}\right), \quad v_{4 T}=\mu_{0}+d_{2 T}+r_{2 c}+\theta_{1}\left(1-r_{2 c}\right), \\
& v_{5 T}=\mu_{0}+k_{r}\left(1-r_{1 r}\right) \quad \text { and } \quad v_{6 T}=\mu_{0}+d_{3 T}+r_{2 r} .
\end{aligned}
$$


The basic reproduction number is defined, following van den Driessche and Watmough [52], as the spectral radius of the next generation matrix, $F V^{-1}$ :

$$
\mathcal{R}_{0}=\max \left\{\mathcal{R}_{H}^{T}, \mathcal{R}_{0}^{H}\right\}
$$

where $\mathcal{R}_{H}^{T}$ and $\mathcal{R}_{0}^{H}$ are the two eigenvalues:

$$
\begin{aligned}
\mathcal{R}_{0}^{H} & =\frac{\beta_{H}\left(A+\eta q \gamma_{1}\right)\left[\pi_{H}\left[\delta(\mu+\Lambda)+\mu \mu_{0}(1-\omega)\right]+\mu_{0}(1-\delta)(\Lambda+\mu \omega)\right]}{A\left(\mu_{0}+\delta\right)\left(\mu_{0}+\gamma_{1}\right)(\Lambda+\mu)}, \\
\mathcal{R}_{H}^{T} & =\frac{\beta_{T}\left[f \mu_{0}+k\left(1-r_{1}\right)\right] V_{0}}{\left(S_{0}+V_{0}\right)\left[\mu_{0}\left(\mu_{0}+d_{T}+r_{2}\right)+k\left(1-r_{1}\right)\left(\mu_{0}+d_{T}\right)\right]}+\frac{(1-\delta) S_{0}}{S_{0}+V_{0}} \mathcal{R}_{0}^{T},
\end{aligned}
$$

with $\mathcal{R}_{0}^{T}$ the basic reproduction number for the TB-only sub-model (3.15).

\section{References}

[1] Global Fund to Fight AIDS, Tuberculosis, and Malaria. Fighting Tuberculosis. Geneva, Switzerland: (2006). Retrieved September 9, 2006, http://www.theglobalfund.org/en/about/tuberculosis/default.asp, 2006.

[2] World Health Organization. Global tuberculosis control: surveillance, planning, financing. Geneva, Switzerland: World Health Organization, 2009.

[3] WHO. Hepatitis B. /http://www.who.int/mediacentre/factsheets/fs204/en/ index.htmlS, revised August 2008, 2008.

[4] C. Dye, B.G. Williams. Eliminating human tuberculosis in the twenty-first century. J. R. Soc. Interface, 5 (2008), 653-662.

[5] C. Chintu, A. Mwinga. An African perspective of tuberculosis and HIV/AIDS. Lancet, 353 (1999), 997-1005.

[6] R. Williams. Global challenges in liver disease. Hepatol., 44 (2006), No. 3, 521-526.

[7] T. Frieden, R.C. Driver. Tuberculosis control: past 10 years and future progress. Tuberculosis, 83 (2003), 82-85.

[8] K.M. De Cock, R.E. Chaisson. Will DOTS do it? A reappraisal of tuberculosis control in countries with high rates of HIV infection. Int. J. Tuberc. Lung Dis., 3 (1999), 457-465.

[9] Global Fund Against AIDS, TB and Malaria. The Global Tuberculosis Epidemic, Geneva, Switzerland, 2004.

[10] D. Lavanchy. Hepatitis B virus epidemiology, disease burden, treatment and current and emerging prevention and control measures. J. Viral. Hepat., 11 (2004), 97-107. 
[11] W.J. Edmunds, G.F. Medley, D.J. Nokes. The transmission dynamics and control of hepatitis $B$ virus in the Gambia. Stat. Med., 15 (1996), 2215-2233.

[12] W.J. Edmunds, G.F. Medley, D.J. Nokes. Vaccination against hepatitis B virus in highly endemic area: waning vaccine-induced immunity and the need for booster doses. Trans. R. Soc. Trop. Med. Hyg., 90 (1996), 436-440.

[13] W.J. Edmunds, G.F. Medley, D.J. Nokes, A.J. Hall, H.C. Whittle. The influence of age on the development of the hepatitis B carrier state. Proc. R. Soc. Lond. B, 253 (1993), 197-201.

[14] W.J. Edmunds, G.F. Medley, D.J. Nokes, A.J. Hall, H.C. Whittle. Epidemiological patterns of hepatitis B virus (HBV) in highly endemic areas. Epidemiol. Infect., 117 (1996), 313-325.

[15] S.T. Goldstein, F.J. Zhou, S.C. Hadler, B.P. Bell, E.E. Mast, H.S. Margolis. A mathematical model to estimate global hepatitis $B$ disease burden and vaccination impact. Int. J. Epidemiol., 34 (2005), 1329-1339.

[16] S. Hahnea, M. Ramsaya, K. Balogun, W.J. Edmund, P. Mortimer. Incidence and routes of transmission of hepatitis B virus in England and Wales, 1995-2000:implications for immunisation policy. J. Clin. Virol., 29 (2004), 211-220.

[17] J. Hou, Z. Liu, F. Gu. Epidemiology and prevention of hepatitis B virus infection. Int. J. Med. Sci., 2 (2005), No. 1, 50-57.

[18] K.C. Hyams. Risks of chronicity following acute hepatitis B virus infection: a review. Clin. Infect. Dis., 20 (1995), 992-1000.

[19] J.D. Jia, H. Zhuang. The overview of the seminar on chronic hepatitis B. Chin. J. Hepatol., 12 (2004), 698-699.

[20] D. Lavanchy. Hepatitis B virus epidemiology, disease burden, treatment and current and emerging prevention and control measures. J. Viral. Hepat., 11 (2004) 97-107.

[21] C.A. Blal, S.R.L. Passos, C. Horn, I. Georg, M.G. Bonecini, V.C. Rolla, L. De Castro. High prevalence of hepatitis B virus among tuberculosis patients with and without HIV in Rio de Janeiro, Brazil. Eur. Soc. Clin. Micro., 24 (2005), 41-43.

[22] M.H. Kuniholm, J. Mark, M. Aladashvili, N. Shubladze, G. Khechinashvili, T. Tsertsvadze, C. del Rio, K.E. Nelson. Risk factors and algorithms to identify hepatitis $C$, hepatitis B, and HIV among Georgian tuberculosis patients. Int. Soc. Inf. Dis., (2007) doi:10.1016/j.ijid.2007.04.015.

[23] R. Bellamy, C. Ruwende, T. Corrah, K.P.W.J. McAdam, M. Thursz, H.C. Whittle, A.V.S. Hill. Tuberculosis and Chronic Hepatitis B Virus Infection in Africans and Variation in the Vitamin D Receptor Gene. J. Inf. Dis., 179 (1999), 721-724. 
[24] A.R. Lifson, D. Thai, A. O'Fallon, W.A. Mills, K. Hang. Prevalence of tuberculosis, hepatitis $B$ virus, and intestinal parasitic infections among refugees to Minnesota. Public Health Rep., 117 (2002), 69-77.

[25] K.A. McGlynn, E.D. Lustbader, W.T. London. Immune responses to hepatitis B virus and tuberculosis infections in Southeast Asian refugees. Amer. J. Epide., 122 (1985), 1032-1036.

[26] P.A. Patel, M.D. Voigt. Prevalence and interaction of hepatitis $B$ and latent tuberculosis in Vietnamese immigrants to the United States. Amer. J. Gastr., 97 (2002), 1198-1203. doi:10.1111/j.1572-0241.2002.05704.x.

[27] N.W.Y. Leung. Treatment Of Tuberculosis In Patients With Hepatitis. Hong Kong Practitioner, 19 (1997), 6-13.

[28] W.O. Kermack, A.G. McKendrick. A contribution to the mathematical theory of epidemics. Proc. Roy. Soc., A115 (1927), 700-721.

[29] R.M. Anderson, R.M. May. Infectious Disease of Humans: Dynamics and Control. Oxford University Press, London/New York, 1992.

[30] K.B. Blyuss, Y.N. Kyrychko. On a basic model of a two-disease epidemic. Appl. Math. Comput., 160 (2005), 177-187.

[31] R. Naresh, A. Tripathi. Modelling and analysis of HIV-TB co-infection in a variable size population. Math. Model. Anal., 10(3) (2005), 275-286.

[32] E.F. Long, N.K. Vaidya, M.L. Brandeau. Controlling Co-epidemic: Analysis of HIV and tuberculosis infection analysis. Oper. Res., 56 (2008), No. 6, 1366-1381. doi:10.1287/opre.1080.0571.

[33] N. Bacaer, R. Ouifki, C. Pretorious, R. Wood, B. William. Modelling the joint epidemics of TB and HIV in a South African township. J. Math. Biol., 57 (2008), 557-593.

[34] O. Sharomi, C.N. Podder, A.B. Gumel, B. Song. Mathematical analysis of the transmission dynamics of HIV/TB co-infection in the presence of treatment. Math. Biosci. Eng., 5 (2008), 145-174.

[35] Z. Mukandavire, A.B. Gumel, W. Garira, J.M. Tchuenche. Mathematical analysis of a model for HIV-malaria co-infection. Math. Biosci. Engr., 6 (2009), 333-362.

[36] E. Mtisi, H. Rwezaura, J.M. Tchuenche. A mathematical analysis of malaria and tuberculosis co-dynamics. Dis. Cont. Dyn. Syst. Series B, 12 (2009) 827-864 2009doi:10.3934/dcdsb.2009.12.827

[37] L-I.W. Roeger, Z. Feng, C.C. Chavez. Modelling TB and HIV co-infections. Math. Bios. Eng., 6 (2009), 815-837. 
[38] S. Bowong, J.J. Tewa. Mathematical analysis of a tuberculosis model with differential infectivity. Com. Non. Sci. Num. Sim., 14 (2009), 4010-4021.

[39] S. Hahnea, M. Ramsaya, K. Balogun, W.J. Edmund, P. Mortimer. Incidence and routes of transmission of hepatitis B virus in England and Wales, 1995-2000: implications for immunization policy. J. Clin. Virol., 29 (2004), 211-220.

[40] C. Dye, S. Schele. For the WHO global surveillance and monitoring project. Global burden of tuberculosis estimated incidence, prevalence and mortality by country. 282 (1999), 677686.

[41] National Committee of Fight Against Tuberculosis. Guide de personnel de la santé, Cameroon, 2008.

[42] National Institute of Statistics. Evolution des systèmes statistiques nationaux, Cameroon, 2007.

[43] G. Birkhoff, G. C. Rota. Ordinary Differential Equations. 4th edition, John Wiley \& Sons, Inc., New York, 1989.

[44] V. Hutson, K. Schmitt. Permanence and the dynamics of biological systems. Math. Biosci., 111 (1992), 1-71

[45] H.W. Hethcothe. The mathematics of infectious disease. SIAM Review, 42 (2000), 599-653.

[46] C.W. Shepard, E.P. Simard, L. Finelli, A.E. Fiore, B.P. Bell. Hepatitis B virus infection: epidemiology and vaccination. Epidemiol. Rev., 28 (2006), 112-125.

[47] V. Lakshmikantham, S. Leela, A. Martynyuk. Stability Analysis of Nonlinear Systems. Marcel Dekker Inc., New York and Basel, pp. 31, 1989.

[48] H.L. Smith, P. Waltman. The Theory of the Chemostat. Cambridge University Press, 1995.

[49] S.N. Zhang. Comparison theorems on boundedness. Funkcial. Ekvac., 31 (1988), 179-196.

[50] S.M. Moghadas. Modelling the effect of imperfect vaccines on disease epidemiology. Dis. Cont. Dynam. Syst. Series B, 4 (2004), 999-1012.

[51] O. Diekmann, J.A.P. Heesterbeek, J.A.P. Metz. On the definition and computation of the basic reproduction ratio $R_{0}$ in the model of infectious disease in heterogeneous populations. J. Math. Biol., 2 (1990), 265-382.

[52] P. van den Driessche, J. Watmough. Reproduction numbers and sub-threshold endemic equilibria for compartmental models of disease transmission. Math. Bios., 180 (2002), 29-28.

[53] J.P. LaSalle. The stability of dynamical systems. Society for Industrial and Applied Mathematics, Philadelphia, Pa, 1976. 
[54] J.P. LaSalle. Stability theory for ordinary differential equations. J. Differ. Equ., 41 (1968), 57-65.

[55] N.P. Bhatia, G.P. Szegö. Stability Theory of Dynamical Systems. Springer-Verlag, 1970.

[56] J. Carr. Applications Centre Manifold Theory. Springer-Verlag, New York, 1981.

[57] C. Castillo-Chavez, B. Song. Dynamical models of tuberculosis and their applications. Math. Bios. Eng., 1 (2004), 361-404.

[58] J. Dushoff, W. Huang, C. Castillo-Chavez. Backwards bifurcations and catastrophe in simple models of fatal diseases. J. Math. Biol., 36 (1998), 227-248.

[59] J. Arino, C.C. McCluskey, P. van den Driessche . Global result for an epidemic model with vaccination that exihibits backward bifurcation. J. Appl. Math., 64 (2003), 260-276.

[60] F. Brauer. Backward bifurcation in simple vaccination models. J. Math. Ana. Appl., 298 (2004), 418-431.

[61] Z. Feng, C. Castillo-Chavez, A.F. Capurro. A model for tuberculosis with exogenous reinfection. Theor. Pop. Biol., 57 (2000), 235-247.

[62] C.Y. Chiang, L.W. Riley. Exogenous reinfection in tuberculosis. Lancet Infect. Dis., 5 (2005), 629-636.

[63] S.M. Garba, A.B. Gumel, M.R. Abu Bakar. Backward bifurcation in dengue transmission dynamics. Math. Bios., 215 (2008), 11-25.

[64] O. Sharomi, C.N. Podder, A.B. Gumel, E.H. Elbasha, J. Watmough. Role of incidence function in vaccine-induced backward bifurcation in some HIV models. Math. Biosci., 210 (2007), 436-463.

[65] F. Brauer, C. Castillo-Chavez. Mathematical Models in Population Biology and Epidemiology. Text in Applied Mathematics Series, 40, Springer-Verlag, New York, 2001.

[66] B.M. Murphy, B.H. Singer, D. Kirschner. Comparing epidemic tuberculosis in demographically distinct populations. Maths. Biosci., 180 (2002), 161-185. 